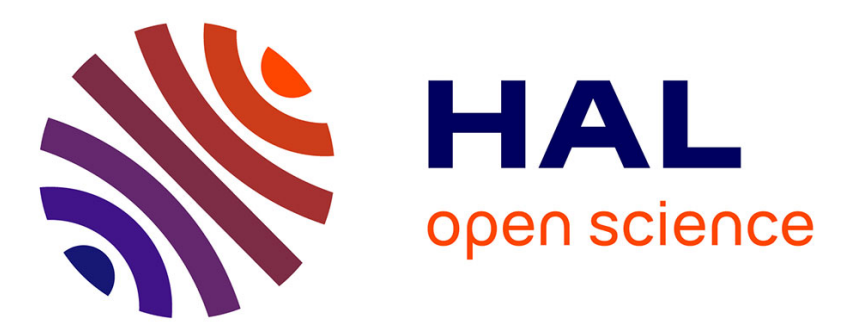

\title{
Measuring the Noise of Digital Imaging Sensors by Stacking Raw Images Affected by Vibrations and Illumination Flickering
}

Frédéric Sur, Michel Grediac

\section{- To cite this version:}

Frédéric Sur, Michel Grediac. Measuring the Noise of Digital Imaging Sensors by Stacking Raw Images Affected by Vibrations and Illumination Flickering. SIAM Journal on Imaging Sciences, 2015, 8 (1), p. 611-643. 10.1137/140977035. hal-01133358

\section{HAL Id: hal-01133358 \\ https://inria.hal.science/hal-01133358}

Submitted on 19 Mar 2015

HAL is a multi-disciplinary open access archive for the deposit and dissemination of scientific research documents, whether they are published or not. The documents may come from teaching and research institutions in France or abroad, or from public or private research centers.
L'archive ouverte pluridisciplinaire HAL, est destinée au dépôt et à la diffusion de documents scientifiques de niveau recherche, publiés ou non, émanant des établissements d'enseignement et de recherche français ou étrangers, des laboratoires publics ou privés. 


\title{
Measuring the Noise of Digital Imaging Sensors by Stacking Raw Images Affected by Vibrations and Illumination Flickering*
}

\author{
Frédéric Sur ${ }^{\dagger}$ and Michel Grédiac ${ }^{\ddagger}$
}

\begin{abstract}
This paper discusses camera noise estimation from a series of raw images of an arbitrary natural static scene, acquired with the same camera settings. Although it seems natural to characterize noise from the random time fluctuation of pixel intensity, it turns out that these fluctuations may also be caused by illumination flickering and mechanical microvibrations affecting the camera. In this context, the contributions are twofold. First, a theoretical model of image formation in the presence of illumination flickering and of vibrations is discussed. This parametric model is based on a Cox process. It is shown that illumination flickering changes the standard affine relation between noise variance and average intensity to a quadratic relation. Second, under these conditions an algorithm is proposed to estimate the main parameters governing sensor noise, namely the gain, the offset, and the readout noise. The rolling shutter effect, which potentially affects the output of any focal-plane shutter camera, is also considered. Experiments show that this simple method gives results consistent with the photon transfer method, which needs a special experimental setting and several data acquisitions, and with an algorithm based on a single image. The main practical result is to show that flickering, which is generally considered as an artifact, here plays a positive role since it finally enables us to estimate any of the sensor parameters.
\end{abstract}

Key words. sensor noise measurement, Poisson-Gaussian noise model, illumination flickering, Cox process, rolling shutter effect

AMS subject classifications. 68U10, 94A08, 62M40, 62H35

DOI. $10.1137 / 140977035$

1. Introduction. Noise is an undesired yet unavoidable feature of digital imaging sensors. Since consumer cameras are expected to offer the most pleasing-to-the-eye images, denoising algorithms have become crucial parts of the image processing chain. Recent papers such as [31] and [38] argue that an accurate modeling of the noise is important for these algorithms. Not only is it needed in denoising applications, but it is also of utmost importance for assessing the metrological performance of contactless measurement systems which spread quickly in various fields of engineering. For example, in experimental mechanics, assessing the noise level in displacement and strain maps obtained with such measurement systems and establishing its link with camera sensor noise is a key issue, as illustrated by recent papers (see, e.g., [22, 24, 39]). Although the Gaussian white noise assumption is widespread, this rough model is inadequate when considering metrological performance assessment or state-of-the-art denoising applications. In the case of raw data from a CCD or CMOS sensor, the authors of [23] identify four

\footnotetext{
* Received by the editors July 10, 2014; accepted for publication (in revised form) January 15, 2015; published electronically March 18, 2015. This research was partially funded by GdR CNRS ISIS (TIMEX project).

http://www.siam.org/journals/siims/8-1/97703.html

${ }^{\dagger}$ Loria, UMR 7503, CNRS, INRIA projet Magrit, Université de Lorraine, BP 239, 54506 Vandoeuvre-lès-Nancy cedex, France (frederic.sur@loria.fr).

‡Institut Pascal, UMR 6602 CNRS, BP 10448, 63171 Aubière cedex, France, and Université Blaise Pascal, BP 185, 63000 Clermont-Ferrand, France (michel.grediac@univ-bpclermont.fr).
}

611

Copyright (C) by SIAM. Unauthorized reproduction of this article is prohibited. 
major sources of noise, namely residual dark current (depending on the ambient temperature and on the exposure time), shot noise (modeling the uncertainty in the electron count at a photosite), readout noise (due to on-chip output amplifier), and quantization noise. The raw output of a linear camera (i.e., the raw sensor output, without any image processing) is a function of the quantity of light photons arriving at a CCD sensor cell, but is affected by shot noise, a dark current, and readout noise. The following stochastic model (or slight variations) is often used $[2,7,18,21,23]$, yielding a heteroscedastic signal-dependent noise:

$$
u(x, y)=g \eta_{p(x, y)+d(x, y)}(x, y)+\delta(x, y),
$$

where

- $u(x, y)$ is the intensity or gray level measured at the photosite corresponding to the pixel $(x, y)$ in the raw output image;

- $g>0$ is the gain of the electronic system;

- the number of generated electrons $\eta_{p(x, y)+d(x, y)}$ at $(x, y)$ is a random variable following a Poisson distribution of mean value $p(x, y)+d(x, y)$, assumed to be spatially independently distributed. Here $p(x, y)$ is the number of electrons produced by a part of the incident photons (the proportion depends on the quantum efficiency of the device at a given wavelength) and $d(x, y)$ is the number of "dark" electrons generated during the exposure time;

- $\delta(x, y)$ is a Gaussian white noise of mean $\mu$ (fixed offset value imposed by the sensor manufacturer) and variance $\sigma^{2}$ (caused by readout and quantization noise);

- the random variables $\eta_{p(x, y)+d(x, y)}(x, y)$ and $\delta(x, y)$ are independent.

In this formulation, the limited range (caused by the finite capacity of each photosite) and the quantized nature of the gray-level $u$ are ignored.

In the usual experimental setting (usual temperature and exposure time equal to a fraction of a second) the dark current is negligible relative to the photo-electron count $[2,23]$. In the remainder of this paper, we set $d=0$. In addition, most color camera sensors are actually equipped with a Bayer filter mosaic. In this case, (1.1) holds for each of the color channels.

Within the model of (1.1), the expectation and the variance of any Poisson variable being equal, it is possible to compute $[7,16,18,21,43]$

$$
\left\{\begin{array}{l}
E(u(x, y))=g p(x, y)+\mu \\
\operatorname{Var}(u(x, y))=g^{2} p(x, y)+\sigma^{2}
\end{array}\right.
$$

where $E$ and Var denote, respectively, the expectation and the variance. The following affine relation consequently holds:

$$
\operatorname{Var}(u(x, y))=g E(u(x, y))+\sigma^{2}-g \mu .
$$

Estimating the whole set of noise parameters can be achieved by the so-called "photon transfer method" [47, 25] which needs a controlled experimental setting and several data acquisitions. Estimating the slope $g$ and the intercept $\sigma^{2}-g \mu$ in (1.3) is sufficient for many applications. For instance, the first task of many image denoising algorithms is to stabilize the variance using, e.g., the generalized Anscombe transform [36, 40] (after [3]) which only

Copyright ( ) by SIAM. Unauthorized reproduction of this article is prohibited. 
needs these two parameters. Another application to camera model identification is presented in [54]. In order to estimate the slope and the intercept in (1.3), a simpler method than the photon transfer method is sought. This is the goal of several papers, where these parameters are estimated by using a single image. The sample mean and variance can be evaluated on homogeneous image regions $[16,21,43]$, or a selection of small homogeneous patches $[7,33]$, which is likely to fail with a cluttered scene giving highly textured images. In this case, the authors of [55] model image textures as two-dimensional (2D) fractional Brownian motions and propose an estimator of the signal-dependent noise affecting such textures. The approach of [21] has also been made more robust to high-frequency textures in [4]. A joint estimation of the noise parameters and of an implicit segmentation is proposed in [28]. The authors of [5] eliminate the selection of homogeneous patches by modeling arbitrary patches as Gaussian mixtures. While the above-mentioned papers are mainly based on parametric regressions of variance against expectation plots, a recent approach consists of estimating sensor parameters so that a variance stabilization function performs at its best, as in [29] under the Poisson assumption or as in [37, 42] under the Poisson-Gaussian assumption. Nonparametric approaches based on the estimation of a noise level function of arbitrary shape are also available (see, e.g., [32] and the more recent [11]). These latter approaches are not within the scope of this paper, which is focused on parametric estimation.

Instead of using spatial statistics of a single image, it is also possible to use temporal statistics of a series of images. An image stacking approach would simply consist of taking a series of images of a static scene at different times with the same camera settings, and of computing afterward the sample mean and variance at a given pixel, as in [20]. It turns out, however, that the random fluctuations of the stacked pixel intensities are not solely the result of the digital noise modeled by (1.1), as illustrated in Figure 1. First, even if the camera is fixed to a heavy tripod, mechanical microvibrations are likely to affect the sensor, and hence the imaged scene. It is well known that concrete floor slabs on which cameras or imaged objects rest are prone to vibrations [17]. This phenomenon is very difficult to avoid without a costly dedicated setting such as a vibration insulated optical table or an optical image stabilization system. For example, it has been demonstrated in [57] that the pixel intensity fluctuations along edges of digitized aerial images are temporally correlated because of vibrations during the scanning process. The movement of the mirror or of the shutter in a single-lens reflex (SLR) camera, or the cooling device in a high-end camera may also cause vibrations. Atmospheric turbulence is another potential source of instability of the imaged scene. Even if these perturbations yield motions with a tiny amplitude, they are all the more noticeable as the photosite density is high or the focal length is long. Another important external source of pixel intensity fluctuations is the flickering of the illumination source. A number of things may cause this phenomenon, among which is the domestic alternative current supplying artificial lights [10]. Certain light sources are particularly affected, such as neon tube lights [35], or poorly designed LEDs [1]. A slight variability of the exposure time caused by mechanical or electronic imperfections of the shutter would cause an equivalent effect. Interestingly, a similar deterministic phenomenon can be noticed in fluorescence imaging: an exponential decay of the photon count caused by photobleaching is considered in [27], where the model parameters are estimated using an iterative expectation-minimization approach. In the context of denoising from a burst of images, the authors of [9] discovered that the bursts

Copyright (c) by SIAM. Unauthorized reproduction of this article is prohibited. 

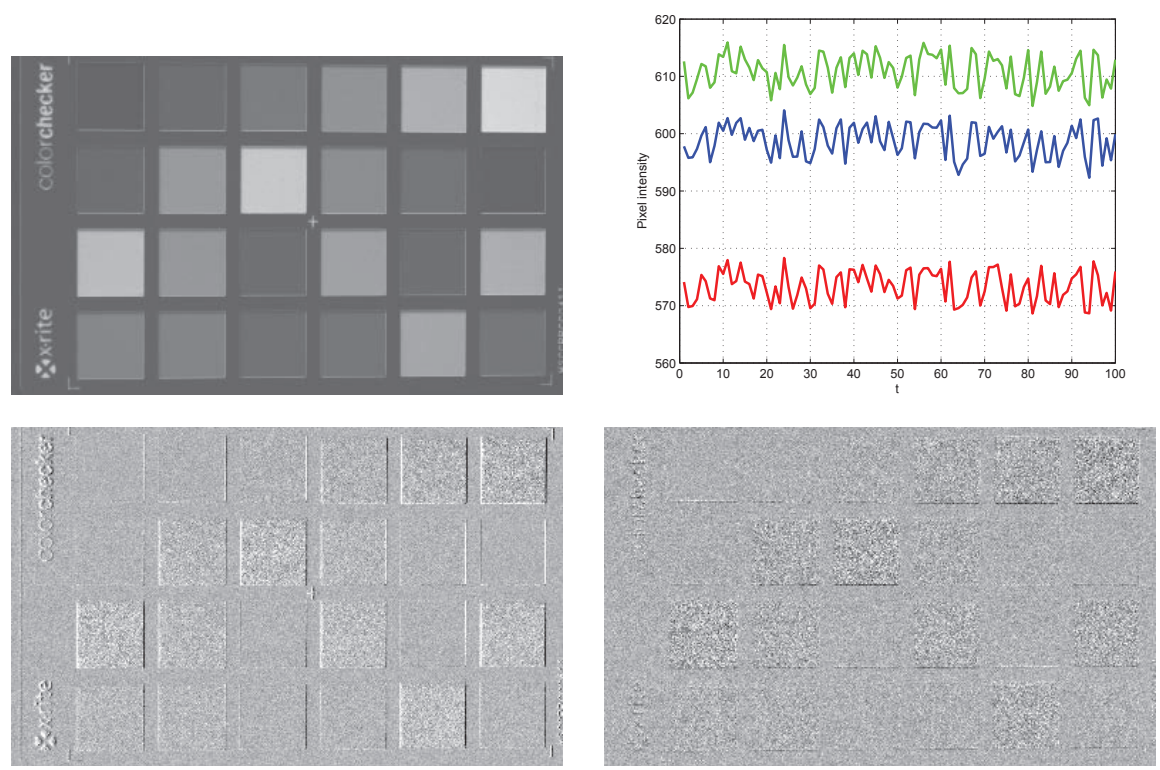

Figure 1. An X-Rite Colorchecker, illuminated by a neon light, is imaged by a Canon EOS 350D camera (ISO 100) equipped with a $50 \mathrm{~mm}$ lens and fixed to a tripod. A burst of 100 images is taken. Top left: A raw image (red channel) from the series. Top right: An average pixel intensity is computed over 100 pixels belonging to the same color patch in order to remove the random digital noise. The graph depicts the evolution of this average intensity at three distant locations belonging to different color patches. In-phase fluctuations can be seen; they are due to light flickering. Bottom: the two images correspond to two examples of a difference between two distant images of the burst (same gray value scale). In the ideal vibration-free case, the difference image would only consist of a noise proportional to the pixel intensity. Here, edges can be seen and are more or less pronounced according to the direction and the gray-level difference between a patch and the black background color. They are caused by mechanical microvibrations.

suffer from changes in the illumination conditions, which makes it impossible to estimate the noise level from intensity changes along time. They circumvent this problem by histogram equalization [14]. Although this method proved to be effective for image denoising, such an approach does not give any guarantee in the estimated noise parameters and thus cannot be used in a metrological framework.

In the end, mechanical vibrations and illumination flickering prevent the sample mean and the sample variance estimated by time-averaging from satisfying the affine relation given by (1.3). The effect of the sole vibrations on noise measurement has been investigated in the case of pseudoperiodic grid images $[50,53]$. This was motivated by the characterization of the metrological performances of the grid method $[22,51]$ in experimental solid mechanics. A short discussion of the flickering effect on noise measurement is available in [49]. Additional information and experiments are available in a separate research report [52].

Contribution and organization of the paper. The contribution of this paper is to investigate the effect of mechanical vibrations and light flickering on the estimation of the noise parameters using image stacking. A theoretical model is proposed in section 2, and a practical algorithm is discussed in section 3. The model is based on quite general assumptions. In particular, no periodicity or pseudoperiodicity assumption on the illumination variability

Copyright ( $)$ by SIAM. Unauthorized reproduction of this article is prohibited. 
is needed. The vibrations are assumed to be of limited amplitude (typically smaller than one pixel), and the algorithm does not need to estimate their amplitude from image to image. Motion blur is not considered in this work. Contrary to $[19,21]$, intensity clipping is not taken into account in the model, which also overlooks defective pixels and blooming effect. Clipped data and defective pixels are simply eliminated in a preliminary step. Under a flickering illumination, the variance of the pixel intensity fluctuation is proved to be a quadratic function of the average intensity, instead of the usual affine dependence. Although the methods based on a single image are not affected by these phenomena, the present algorithm, as any stacking method, does not need any explicit segmentation step. We demonstrate that light flickering makes it possible to estimate all the sensor parameters, namely the gain, offset, and readout noise level. Although very accurate estimations of these parameters (apart from nonuniformity problems) can only be achieved by the photon transfer method, we show that stacking 100 images affected by flickering is often sufficient to get a reasonable estimation. In addition, we mention a workaround to deal with the rolling shutter effect, which especially affects cameras with a focal-plane shutter, such as SLR cameras. The estimation process is evaluated on synthetic and real data (raw images from two SLR cameras and a high-end CCD camera) in section 4. As a sanity check, the process is also compared with the photon transfer method and to the software implementing [21], which only needs a single image. We conclude with section 5 .

MATLAB code implementing the algorithm of section 3 is available online at http://www. loria.fr/\%7Esur/software/NESIF/.

2. Sensor noise modeling in the presence of a flickering illumination and vibrations. The aim of this section is to model the measured intensity at a pixel along time, under a flickering illumination and mechanical vibrations affecting the experimental setup, and to link the time variance to the expected intensity.

2.1. Modeling raw data from a linear camera. A series of $T$ images of a static scene is assumed to be available. With the number of emitted photo-electrons at a given photosite being proportional to the number of incoming photons (through the quantum efficiency), the average number of photo-electrons at a photosite of the $t$ th image can be expressed as $\left(1+\gamma_{t}\right) p\left(x+\alpha_{t}, y+\beta_{t}\right)$, where

- $\left(\alpha_{t}, \beta_{t}\right)$ is the translation vector modeling the sensor in-plane displacement due to vibrations between an unknown reference image and the $t$ th image, and

- $1+\gamma_{t}$ is the relative fluctuation of the illumination intensity.

It should be noted that motion blur, which would require an integration of $p$ along time instead of a pure translation, is not within the model.

Within the assumptions of section 1 , the intensity $u(x, y, t)$ at pixel $(x, y)$ of the $t$ th image is modeled by

$$
u(x, y, t)=g \eta_{\left(1+\gamma_{t}\right) p\left(x+\alpha_{t}, y+\beta_{t}\right)}(x, y, t)+\delta(x, y, t),
$$

where the random variables $\eta$ and $\delta$ are time-independent and spatially distributed as above. Adding constant terms to $x$ and $y$ means that we only consider translations, which corresponds to what is observed in practice in all the experiments described in section 4 below.

Copyright $\odot$ by SIAM. Unauthorized reproduction of this article is prohibited. 
Both processes $\gamma_{t}$ and $\left(\alpha_{t}, \beta_{t}\right)$ are modeled as an independently and identically distributed 0 -mean random process. The variance of $\gamma$ is noted $\sigma_{\gamma}^{2}$ and the $2 \times 2$ covariance matrix of $\left(\alpha_{t}, \beta_{t}\right)$ is $\operatorname{Cov}(\alpha, \beta)$.

In this model, the intensity of the Poisson variable is also a random process. It is called a mixed doubly stochastic Poisson process, or Cox process, after [12]. The intensity $u(x, y, t)$ is thus a realization of a Cox-Gaussian process. The goal of the following section is to calculate a relation between the variance of $u$ and its expectation.

2.2. Noise variance against intensity expectation. In the following calculations, we make use of the law of the total expectation and of the law of the total variance: if $X$ is an integrable random variable and $Y$ is another random variable, then $E(X)=E(E(X \mid Y))$ and $\operatorname{Var}(X)=E(\operatorname{Var}(X \mid Y))+\operatorname{Var}(E(X \mid Y))$. We also recall that if $X$ and $Y$ are two independent random variables, then $E(X Y)=E(X) E(Y)$. For conciseness, we skip the mention of $(x, y, t)$ after $u$ and $\delta$, and of $(x, y)$ after $p$.

Let $\eta_{\lambda}$ be a Cox process: this means that, conditional on $\lambda=l, \eta_{l}$ is a Poisson process of mean value $l$. With the law of the total expectation and the law of the total variance, we obtain

$$
\left\{\begin{array}{l}
E\left(\eta_{\lambda}\right)=E\left(E\left(\eta_{\lambda} \mid \lambda\right)\right)=E(\lambda) \\
\operatorname{Var}\left(\eta_{\lambda}\right)=E\left(\operatorname{Var}\left(\eta_{\lambda} \mid \lambda\right)\right)+\operatorname{Var}\left(E\left(\eta_{\lambda} \mid \lambda\right)\right)=E(\lambda)+\operatorname{Var}(\lambda)
\end{array}\right.
$$

This expression of the variance means that a Cox process is overdispersed relative to the Poisson process [13]. Under a flickering light and/or vibrations, the time-variance of the intensity at a given pixel thus always overvalues the expected variance given by the standard Poisson-Gaussian model. More precisely, we calculate the following from the properties of the expectation and variance of a Cox process:

$$
\begin{aligned}
E(u) & =E\left(g \eta_{\left(1+\gamma_{t}\right) p\left(\cdot+\alpha_{t}, \cdot+\beta_{t}\right)}+\delta\right) \\
& =g E\left(\left(1+\gamma_{t}\right) p\left(\cdot+\alpha_{t}, \cdot+\beta_{t}\right)\right)+\mu \\
& =g E\left(p\left(\cdot+\alpha_{t}, \cdot+\beta_{t}\right)\right)+\mu
\end{aligned}
$$

(where $\cdot$ denotes either $x$ or $y$ ), since $\gamma_{t}$ and $p\left(\cdot+\alpha_{t}, \cdot+\beta_{t}\right.$ ) are independent, and $E\left(\gamma_{t}\right)=0$. Moreover,

$$
\begin{aligned}
\operatorname{Var}(u)= & \operatorname{Var}\left(g \eta_{\left(1+\gamma_{t}\right) p\left(\cdot+\alpha_{t},+\beta_{t}\right)}+\delta\right) \\
= & g^{2} E\left(\left(1+\gamma_{t}\right) p\left(\cdot+\alpha_{t}, \cdot+\beta_{t}\right)\right) \\
& +g^{2} \operatorname{Var}\left(\left(1+\gamma_{t}\right) p\left(\cdot+\alpha_{t}, \cdot+\beta_{t}\right)\right)+\sigma^{2} \\
= & g^{2} E\left(p\left(\cdot+\alpha_{t}, \cdot+\beta_{t}\right)\right)+g^{2}\left(1+\sigma_{\gamma}^{2}\right) E\left(p^{2}\left(\cdot+\alpha_{t}, \cdot+\beta_{t}\right)\right) \\
& -g^{2}\left(E\left(p\left(\cdot+\alpha_{t}, \cdot+\beta_{t}\right)\right)\right)^{2}+\sigma^{2} .
\end{aligned}
$$

Copyright ( $\odot$ by SIAM. Unauthorized reproduction of this article is prohibited. 
Indeed, with the law of the total variance, we have

$$
\begin{aligned}
\operatorname{Var}\left(\left(1+\gamma_{t}\right) p\left(\cdot+\alpha_{t}, \cdot+\beta_{t}\right)\right)= & E\left(\operatorname{Var}\left(\left(1+\gamma_{t}\right) p\left(\cdot+\alpha_{t}, \cdot+\beta_{t}\right) \mid \alpha_{t}, \beta_{t}\right)\right) \\
& +\operatorname{Var}\left(E\left(\left(1+\gamma_{t}\right) p\left(\cdot+\alpha_{t}, \cdot+\beta_{t}\right) \mid \alpha_{t}, \beta_{t}\right)\right) \\
= & \sigma_{\gamma}^{2} E\left(p^{2}\left(\cdot+\alpha_{t}, \cdot+\beta_{t}\right)\right)+\operatorname{Var}\left(p\left(\cdot+\alpha_{t}, \cdot+\beta_{t}\right)\right) \\
= & \left(1+\sigma_{\gamma}^{2}\right) E\left(p^{2}\left(\cdot+\alpha_{t}, \cdot+\beta_{t}\right)\right) \\
& -\left(E\left(p\left(\cdot+\alpha_{t}, \cdot+\beta_{t}\right)\right)\right)^{2}
\end{aligned}
$$

since $\operatorname{Var}\left(p\left(\cdot+\alpha_{t}, \cdot+\beta_{t}\right)\right)=E\left(p^{2}\left(\cdot+\alpha_{t}, \cdot+\beta_{t}\right)\right)-\left(E\left(p\left(\cdot+\alpha_{t}, \cdot+\beta_{t}\right)\right)\right)^{2}$, and hence we have (2.8).

As we can see from (2.5) and (2.8), the term $E\left(p^{2}\left(x+\alpha_{t}, y+\beta_{t}\right)\right)$ prevents us from writing $\operatorname{Var}(u)$ as a simple function of $E(u)$. Nevertheless, inspired by the Delta-method [41] we can use a second-order Taylor series expansion,

$$
\begin{aligned}
p\left(x+\alpha_{t}, y+\beta_{t}\right)= & p(x, y)+\left(\alpha_{t}, \beta_{t}\right) \nabla p(x, y) \\
& +\frac{1}{2}\left(\alpha_{t}, \beta_{t}\right) H_{p}(x, y)\left(\alpha_{t}, \beta_{t}\right)^{T}+R_{p}\left(x, y, \alpha_{t}, \beta_{t}\right)
\end{aligned}
$$

with $\nabla$ the gradient, $H_{p}(x, y)$ the Hessian matrix of $p$ at $(x, y)$, and the remainder $R_{p}$ being a multivariate polynomial in $\left(\alpha_{t}, \beta_{t}\right)$ with monomials of total degree larger than 3.

On the one hand, taking first the square of (2.12), then the expectation, we get

$$
\begin{aligned}
E\left(p^{2}\left(\cdot+\alpha_{t}, \cdot+\beta_{t}\right)\right)=p^{2} & +\nabla p^{T} \operatorname{Cov}(\alpha, \beta) \nabla p \\
& +p E\left(\left(\alpha_{t}, \beta_{t}\right) H_{p}\left(\alpha_{t}, \beta_{t}\right)^{T}\right)+M_{p}^{1}\left(\cdot, \cdot, \alpha_{t}, \beta_{t}\right),
\end{aligned}
$$

where $M_{p}^{1}$ is a linear combination of mixed moments of $\left(\alpha_{t}, \beta_{t}\right)$ of order larger than 3 . To establish (2.13), we have used

$$
\begin{aligned}
E\left(\left(\left(\alpha_{t}, \beta_{t}\right) \nabla p\right)^{2}\right)= & E\left(\alpha_{t}^{2}\right)(\partial p / \partial x)^{2}+E\left(\beta_{t}^{2}\right)(\partial p / \partial y)^{2} \\
& +2 E\left(\alpha_{t} \beta_{t}\right)(\partial p / \partial x)(\partial p / \partial y) \\
= & \nabla p^{T} \operatorname{Cov}(\alpha, \beta) \nabla p
\end{aligned}
$$

On the other hand, taking first the expectation of (2.12), then its square

$$
\begin{aligned}
\left(E\left(p\left(\cdot+\alpha_{t}, \cdot+\beta_{t}\right)\right)\right)^{2}= & \left(p+E\left(\frac{1}{2}\left(\alpha_{t}, \beta_{t}\right) H_{p}\left(\alpha_{t}, \beta_{t}\right)^{T}\right)\right. \\
& \left.+E\left(R_{p}\left(\cdot, \cdot, \alpha_{t}, \beta_{t}\right)\right)\right)^{2} \\
= & p^{2}+p E\left(\left(\alpha_{t}, \beta_{t}\right) H_{p}\left(\alpha_{t}, \beta_{t}\right)^{T}\right)+M_{p}^{2}\left(\cdot, \cdot, \alpha_{t}, \beta_{t}\right),
\end{aligned}
$$

where

$$
\begin{aligned}
M_{p}^{2}\left(\cdot, \cdot, \alpha_{t}, \beta_{t}\right)= & \left(E\left(\frac{1}{2}\left(\alpha_{t}, \beta_{t}\right) H_{p}\left(\alpha_{t}, \beta_{t}\right)^{T}\right)+E\left(R_{p}\left(\cdot, \cdot, \alpha_{t}, \beta_{t}\right)\right)\right)^{2} \\
& +2 p E\left(R_{p}\left(\cdot, \cdot, \alpha_{t}, \beta_{t}\right)\right)
\end{aligned}
$$

Copyright (C) by SIAM. Unauthorized reproduction of this article is prohibited. 
is a combination of mixed moments of $\left(\alpha_{t}, \beta_{t}\right)$ of order larger than 3 , of squares of moments of order 2, and of products of moments of order 2 and larger.

With (2.13) and (2.17), we obtain

$$
\begin{aligned}
E\left(p^{2}\left(x+\alpha_{t}, y+\beta_{t}\right)\right) & =\left(E\left(p\left(x+\alpha_{t}, y+\beta_{t}\right)\right)\right)^{2}+\nabla p^{T} \operatorname{Cov}(\alpha, \beta) \nabla p \\
& +M_{p}\left(x, y, \alpha_{t}, \beta_{t}\right)
\end{aligned}
$$

with $M_{p}=M_{p}^{1}-M_{p}^{2}$.

By substituting in $(2.8) g E\left(p\left(\cdot+\alpha_{t}, \cdot+\beta_{t}\right)\right)$ by $E(u)-\mu($ cf. $(2.5))$ and $E\left(\left(p^{2}\left(\cdot+\alpha_{t}, \cdot+\beta_{t}\right)\right)\right.$ by the expression given by (2.19), we eventually obtain

$$
\begin{aligned}
\operatorname{Var}(u)=\sigma_{\gamma}^{2} E(u)^{2}+\left(g-2 \mu \sigma_{\gamma}^{2}\right) E(u)+\sigma^{2}-g \mu+\sigma_{\gamma}^{2} \mu^{2} \\
+\left(1+\sigma_{\gamma}^{2}\right) \nabla E(u)^{T} \operatorname{Cov}(\alpha, \beta) \nabla E(u)+M_{p}\left(x, y, \alpha_{t}, \beta_{t}\right) .
\end{aligned}
$$

Under the mild assumption that the vibrations follow a Gaussian process, moments of order larger than 3 are products of moments of order 2 [26], which justifies that, for small vibrations, the term $M_{p}$ is negligible compared to the term with $\operatorname{Cov}(\alpha, \beta)$.

We have proved that, because of flickering, the affine relation of (1.3) between the variance and the expected pixel intensity transforms into a quadratic relation, and that the vibrations give an additional bias.

At a pixel where the gradient is negligible (or, more generally, where the neighboring pixels have the same intensity), the relation is simply written as

$$
\operatorname{Var}(u)=\sigma_{\gamma}^{2} E(u)^{2}+\left(g-2 \mu \sigma_{\gamma}^{2}\right) E(u)+\sigma^{2}-g \mu+\sigma_{\gamma}^{2} \mu^{2} .
$$

Of course, $\sigma_{\gamma}=0$ simplifies the quadratic expression (2.21) into the affine one given in (1.3). Moreover, even in the flicker-free case, (2.20) shows that the variance estimation is always an overestimation of the expected variance because of the vibrations.

2.3. Discussion. Several properties of the model are now discussed.

2.3.1. Sample mean and sample variance. In the remainder of this paper, the sample mean $1 / T \sum_{t=1}^{T} X(t)$ of any random process $(X(t))_{1<t<T}$ is noted $\bar{X}$. The sample mean $\bar{u}(x, y)$ is an unbiased estimator of $g E\left(p\left(x+\alpha_{t}, y+\beta_{t}\right)\right)+\mu$. Since its variance is equal to $\sum_{t} \operatorname{Var}(u(x, y, t)) / T^{2}=\mathcal{O}(1 / T)$, this estimator is also consistent. ( $\mathcal{O}$ denotes Landau's "bigO".) The unbiased time sample variance $\bar{\sigma}_{u}^{2}(x, y)$ has an expectation equal to $\operatorname{Var}(u(x, y, t))$, and it is well known that its variance decreases as $1 / T$ as soon as the distribution kurtosis is finite. The empirical variance is thus also a consistent estimator of the variance.

Disregarding the vibrations, it should be noted that flickering makes it impossible to estimate the noise parameters $g$ and $\sigma^{2}-g \mu$ with a quadratic regression (because of the $\sigma_{\gamma}^{2}$ term), contrary to the existing parametric methods based on a single image cited in the introduction, where linear regression is sufficient.

2.3.2. Nonstatic scenes. The preceding calculation assumes that the scene is static and that the vibrations equally affect all the sensor pixels. Nevertheless, it is common for an outdoor scene to have at least some slightly moving parts. For example, the scene may show

Copyright ( $)$ by SIAM. Unauthorized reproduction of this article is prohibited. 
trees swinging because of the wind, or passersby, which would affect the series of images. It should be noted that (2.20) holds locally, that is, the $2 \mathrm{D}$ random process $\left(\alpha_{t}, \beta_{t}\right)$ may depend on $(x, y)$. However, in the case of large movements, the Taylor expansion no longer holds in (2.12). If the nonstatic part of the scene is limited, the experimental results presented here are likely to hold, but, in general, nonstatic scenes are clearly beyond the scope of this paper.

2.3.3. Link with ICCD. It has been shown in [6] (cited by $[44,45]$ ) that a quadratic relation with different parameters also holds when considering an intensified charged couple device (ICCD). The difference with the standard CCD model described in (1.1) is that the gain $g$ is modeled in an ICCD as a random variable. In the present model, the gain is fixed, but the intensity of the Poisson process is a random variable.

2.3.4. Spatial nonuniformity. The standard model of (1.3) assumes that the gain, the quantum efficiency, and the dark noise are uniform. However, manufacturing variability and component tolerances make these quantities vary from pixel to pixel, yielding the so-called dark signal nonuniformity (DSNU) and photo response nonuniformity (PRNU) [47, 23, 25]. These departures from ideality amount in (1.3) (and in the following calculations) to considering $\gamma_{t}$, $g$, and $\delta$ as functions of $(x, y)$, in order to take into account the nonuniformity of, respectively, quantum efficiency, gain, and dark signal.

This means that the scatter plots of time sample variance against time sample mean shall show a variability caused not only by the usual variability of any sample estimator but also by DSNU and PRNU. The authors of [44] observe gradual spatial nonuniformity in the case of ICCD and design a parametric model to deal with it. In CCD or CMOS, it is known that PRNU gives an additional intensity variability up to some percent $[46,47]$. The effect on the quadratic relation caused by flickering is all the more important because of the quadratic term.

In the remainder of this article, we do not take PRNU and DSNU into account, as do the papers cited in the introduction. Nevertheless, these phenomena explain a part of the variability in the expected relations.

3. Estimating noise parameters. It is in fact possible to remove the effect of light flickering in the variance estimation. The scene is indeed static and shows no moving object (apart from the microvibrations), and the flickering is assumed to be (until section 3.5) uniform over each image, contrary to the more complicated classic flicker removal problem in movies; see, e.g., [15]. The proposed algorithm to estimate noise parameters consists of the following steps:

1. Use a statistical test to eliminate the pixels where the gradient is too large to be caused by random noise, and keep only those pixels such that (2.21) is satisfied. This preprocessing step permits us to eliminate the bias caused by the vibrations. Clipped or defective pixels are also eliminated. See section 3.1.

2. Identify the flicker parameter $\gamma_{t}$ for each image of the stack and remove the effect of flicker in the sample variance estimated from the image stack. See section 3.2.

3. Estimate the noise parameters using linear regression on flicker-reduced intensity variation. See section 3.3.

As we shall see, the output of the proposed algorithm is an estimation of all parameters of the linear camera model, namely $g, \mu$, and $\sigma$, and not only of the parameters $g$ and $\sigma^{2}-g \mu$

Copyright $\odot$ by SIAM. Unauthorized reproduction of this article is prohibited. 
of the affine relation (1.3).

3.1. Removing pixels affected by physical vibrations or clipping. The relationship given by (2.20) shows that physical vibrations bias the estimation of the pixel intensity variance as a function of the gradient of the expected intensity. In order to remove the effects of the vibrations, we keep only pixels where the local gradient is likely to be due to random noise instead of an actual edge. As the gradient is being evaluated here with a centered difference scheme, its squared Euclidean norm is

$$
\begin{aligned}
\|\nabla \bar{u}(x, y)\|^{2}= & (\bar{u}(x+1, y)-\bar{u}(x-1, y))^{2} / 4 \\
& +(\bar{u}(x, y+1)-\bar{u}(x, y-1))^{2} / 4 .
\end{aligned}
$$

Under the hypothesis that $E(u)$ is constant in the 4-neighborhood of $(x, y)$ and that pixel intensities are independently distributed, $(\bar{u}(x+1, y)-\bar{u}(x-1, y)) / 2$ has 0-mean and variance $\operatorname{Var}(u(x, y, t)) /(2 T)$. Therefore, under a Gaussian distribution assumption, $2 T\|\nabla \bar{u}\|^{2} / \operatorname{Var}(u(x, y, t))$ follows a $\chi^{2}$ law with two degrees of freedom.

We consequently eliminate pixels such that the $p$-value of $2 T\|\nabla \bar{u}\|^{2} / \bar{\sigma}_{u}^{2}$ under this probability distribution is below 1\%; that is, the former quantity must be smaller than 9.21 to keep the pixels.

We eventually discard pixels of coordinates $(x, y)$ as soon as one of the intensity values in the time series $u(x, y, t)$ equals the lowest or highest possible gray-level output (the range being $2^{b}$, where $b$ is the bit-depth). This procedure removes clipped pixels and defective pixels (hot or dead photosites).

3.2. Flicker reduction. The proposed flicker reduction procedure consists first in estimating $\gamma_{t}$ for any $t$. The intuition is that $g p(x, y)+\mu$ is much larger than $\mu$ and $\delta$ for large intensity values, enabling further simplification. Indeed, let $v(x, y, t)$ be the ratio between the intensity and the average intensity of a given pixel:

$$
v(x, y, t)=\frac{u(x, y, t)}{\bar{u}(x, y)} .
$$

This simplifies into

$$
v(x, y, t) \simeq \frac{g \eta_{\left(1+\gamma_{t}\right) p(x, y)}+\delta(x, y)}{g p(x, y)+\mu} \simeq \frac{\eta_{\left(1+\gamma_{t}\right) p(x, y)}}{p(x, y)}
$$

which is a random variable of mean $1+\gamma_{t}$ and variance $\left(1+\gamma_{t}\right) / p(x, y) \simeq 1 / p(x, y)$. Consequently, this quantity should give an estimation of $1+\gamma_{t}$.

A more accurate estimation is actually available. If $X$ and $Y$ are random variables, a Taylor expansion of $X / Y$ around $(E(X), E(Y))$ gives the following approximations:

$$
\left\{\begin{array}{l}
E\left(\frac{X}{Y}\right) \simeq \frac{E(X)}{E(Y)}-\frac{\operatorname{Cov}(X, Y)}{E(Y)^{2}}+\frac{E(X) \operatorname{Var}(Y)}{E(Y)^{3}} \\
\operatorname{Var}\left(\frac{X}{Y}\right) \simeq \frac{\operatorname{Var}(X)}{E(Y)^{2}}-\frac{2 E(X) \operatorname{Cov}(X, Y)}{E(Y)^{3}}+\frac{E(X)^{2} \operatorname{Var}(Y)}{E(Y)^{4}}
\end{array}\right.
$$


Here, with the time-independence assumption,

$$
\left\{\begin{array}{l}
E\left(u \mid \gamma_{t}\right)=g\left(1+\gamma_{t}\right) p+\mu \\
\operatorname{Var}\left(u \mid \gamma_{t}\right)=g^{2}\left(1+\gamma_{t}\right) p+\sigma^{2} \\
E\left(\bar{u} \mid \gamma_{t}\right)=\frac{1}{T} \sum_{t=1}^{T},\left(g\left(1+\gamma_{t}\right) p+\mu\right)=g(1+\bar{\gamma}) p+\mu \\
\operatorname{Var}\left(\bar{u} \mid \gamma_{t}\right)=\frac{1}{T}\left(g^{2}(1+\bar{\gamma}) p+\sigma^{2}\right)=\mathcal{O}(1 / T) \\
\operatorname{Cov}\left(u, \bar{u} \mid \gamma_{t}\right)=\frac{1}{T} \operatorname{Var}\left(u \mid \gamma_{t}\right)=\mathcal{O}(1 / T) .
\end{array}\right.
$$

Since $E\left(\gamma_{t}\right)=0$, we can further simplify with $\bar{\gamma}=\mathcal{O}(1 / T)$. Substituting the expressions (3.5) in (3.4) and keeping only the most influencing terms lead to

$$
\left\{\begin{aligned}
E\left(v \mid \gamma_{t}\right) & =\frac{g\left(1+\gamma_{t}\right) p+\mu}{g(1+\mathcal{O}(1 / T)) p+\mu}+\mathcal{O}(1 / T) \\
& =\frac{1+\gamma_{t}+\mu /(g p)}{1+\mu /(g p)}+\mathcal{O}(1 / T) \\
\operatorname{Var}\left(v \mid \gamma_{t}\right) & =\frac{g^{2}\left(1+\gamma_{t}\right) p+\sigma^{2}}{(g(1+\bar{\gamma}) p+\mu)^{2}}+\mathcal{O}(1 / T) \\
& =\frac{g\left(1+\gamma_{t}\right) E(u)+\sigma^{2}-g\left(1+\gamma_{t}\right) \mu}{E(u)^{2}}+\mathcal{O}(1 / T)
\end{aligned}\right.
$$

We can see from (3.6) that, for large values of the average gray level, $E(u)=g p+\mu$, $E\left(v \mid \gamma_{t}\right)$ is an approximation of $1+\gamma_{t} /(1+\mu /(g p))$ with a minimum variance. Moreover, an estimation of $E\left(v(x, y, t) \mid \gamma_{t}\right)$ is given by averaging $v(x, y, t)$ over a set of pixels for which $\bar{u}(x, y)$ is constant. This motivates the definition of the set

$$
\mathcal{S}^{*}=\left\{(x, y), u^{*}-3 \sigma_{u^{*}} \leq \bar{u}(x, y) \leq u^{*}+3 \sigma_{u^{*}}\right\},
$$

where $u^{*}$ is the $99 \%$ quantile in the empirical intensity distribution of the averaged image $\bar{u}=$ $g p+\mu+\mathcal{O}(1 / T)$, and $\sigma_{u^{*}}$ is the associated standard deviation. We assume that $\bar{u}(x, y, t)$ is constant over $\mathcal{S}^{*}$, equal to $u^{*}$. An estimation of $E\left(v(x, y, t) \mid \gamma_{t}\right)$ is then computed as a sample mean over the set $\mathcal{S}^{*}$.

Indeed, for any $t$,

$$
v^{*}(t)=\frac{1}{\# \mathcal{S}^{*}}\left(\sum_{(x, y) \in \mathcal{S}^{*}} v(x, y, t)\right)
$$

is a random variable of expectation and variance

$$
\left\{\begin{array}{l}
E\left(v^{*}(t)\right)=\frac{1+\gamma_{t}+\mu /\left(u^{*}-\mu\right)}{1+\mu /\left(u^{*}-\mu\right)}+\mathcal{O}(1 / T), \\
\operatorname{Var}\left(v^{*}(t)\right)=\mathcal{O}\left(1 / \# \mathcal{S}^{*}+1 / T \# \mathcal{S}^{*}\right),
\end{array}\right.
$$

where $\# \mathcal{S}^{*}$ denotes the cardinality of $\mathcal{S}^{*}$.

For the sake of simplicity, we skip the mention of $\mathcal{O}(1 / T)$ and $\mathcal{O}\left(1 / \# \mathcal{S}^{*}\right)$ in the remainder of this section. It amounts to assuming that the number of stacked images and the size of $\mathcal{S}^{*}$ are large enough.

Copyright $\odot$ by SIAM. Unauthorized reproduction of this article is prohibited. 
Once $v^{*}(t)$ has been calculated, the quantity defined for any $(x, y, t)$ by $\widetilde{u}(x, y, t)=$ $u(x, y, t)-v^{*}(t) \bar{u}(x, y)$ is such that

$$
\left\{\begin{aligned}
E\left(\widetilde{u}(x, y, t) \mid \gamma_{t}\right)= & g\left(1+\gamma_{t}\right) p(x, y)+\mu \\
& -\left(\frac{1+\gamma_{t}+\mu /\left(u^{*}-\mu\right)}{\left.1+\mu / u^{*}-\mu\right)}\right)(g p(x, y)+\mu) \\
= & g \gamma_{t} p(x, y)-\gamma_{t} \frac{u^{*}-\mu}{u^{*}}(g p(x, y)+\mu) \\
= & -\gamma_{t} \frac{\mu}{u^{*}}\left(u^{*}-\mu-g p(x, y)\right), \\
\operatorname{Var}\left(\widetilde{u}(x, y, t) \mid \gamma_{t}\right)= & g^{2}\left(1+\gamma_{t}\right) p(x, y)+\sigma^{2} .
\end{aligned}\right.
$$

Let $K=E\left(\widetilde{u}(x, y, t) \mid \gamma_{t}\right) /\left(E\left(v^{*}(t)\right)-1\right)$. One has

$$
\begin{aligned}
K & =-\frac{\mu}{u^{*}}\left(u^{*}-\mu-g p(x, y)\right) \frac{u^{*}}{u^{*}-\mu} \\
& =-\mu\left(1-\frac{g p(x, y)}{u^{*}-\mu}\right) .
\end{aligned}
$$

We can see that $K$ does not depend on $t$. For a fixed $p(x, y)$, there is thus a proportionality relation between $E\left(\widetilde{u}(x, y, t) \mid \gamma_{t}\right)$ and $E\left(v^{*}(t)-1\right)$ for every $t$. This proportionality coefficient can be estimated by linear regression. As explained earlier, $E\left(v^{*}(t)\right)$ is obtained by nonlocal estimation over the set $\mathcal{S}^{*}$. Similarly, we choose an iso-value set to estimate, for every $t$, $E\left(\widetilde{u}(x, y, t) \mid \gamma_{t}\right)$. We can see in (3.10) that the smallest value of $\operatorname{Var}\left(\widetilde{u}(x, y, t) \mid \gamma_{t}\right)$ is attained for small $p(x, y)$. We thus define the set

$$
\mathcal{S}_{*}=\left\{(x, y), u_{*}-3 \sigma_{u_{*}} \leq \bar{u}(x, y) \leq u_{*}+3 \sigma_{u_{*}}\right\},
$$

where $u_{*}$ is the $1 \%$ quantile in the intensity distribution of the averaged image $\bar{u}(x, y)$, and $\sigma_{u_{*}}$ is the associated standard deviation. In the same way as $v^{*}$, an estimation of $E\left(\widetilde{u}(x, y, t) \mid \gamma_{t}\right)$ for $(x, y)$ such that $g p(x, y)=u_{*}-\mu$ is given by

$$
v_{*}(t)=\frac{1}{\# \mathcal{S}_{*}}\left(\sum_{(x, y) \in \mathcal{S}_{*}} \widetilde{u}(x, y, t)\right) .
$$

Finally, a linear regression on the scatter plot $\left(v^{*}(t)-1, v_{*}(t)\right)$ gives an estimation of $K$. This permits us to obtain estimations of $\mu$ and $\gamma_{t}$. Indeed, from (3.12),

$$
K=-\mu\left(1-\frac{u_{*}-\mu}{u^{*}-\mu}\right),
$$

hence $K\left(u^{*}-\mu\right)=-\mu\left(u^{*}-u_{*}\right)$, and

$$
\mu=\frac{K u^{*}}{K-u^{*}+u_{*}} .
$$

With this estimation of $\mu$, we deduce by identifying $v^{*}$ and its expectation given by (3.9) that

$$
\widetilde{\gamma}(t)=\left(v^{*}(t)-1\right)\left(1+\frac{\mu}{u^{*}-\mu}\right)
$$

Copyright ( $)$ by SIAM. Unauthorized reproduction of this article is prohibited. 
is an estimator of $\gamma_{t}$ for every $t$.

With these estimations of $\gamma_{t}$ and $\mu$, identified in the remainder of the section with their actual values, we can define

$$
\widehat{u}(x, y, t)=u(x, y, t)-\left(1+\gamma_{t}\right)(\bar{u}(x, y)-\mu) .
$$

This quantity is such that

$$
E(\widehat{u})=E\left(E\left(\widehat{u} \mid \gamma_{t}\right)\right)=E(\mu)=\mu
$$

and

$$
\begin{aligned}
\operatorname{Var}(\widehat{u})= & E\left(\operatorname{Var}\left(\widehat{u} \mid \gamma_{t}\right)\right)+\operatorname{Var}\left(E\left(\widehat{u} \mid \gamma_{t}\right)\right) \\
= & E\left(g^{2}\left(1+\gamma_{t}\right) p+\sigma^{2}+\left(1+\gamma_{t}\right)^{2} \operatorname{Var}(\bar{u})\right) \\
& +\operatorname{Var}(\mu) \\
= & g^{2} p+\sigma^{2}+\left(1+\sigma_{\gamma}^{2}\right) \operatorname{Var}(\bar{u}) \\
= & g E(u)+\sigma^{2}-g \mu+\left(1+\sigma_{\gamma}^{2}\right) \operatorname{Var}(\bar{u}(x, y)) .
\end{aligned}
$$

Since $\operatorname{Var}(\bar{u}(x, y))=\mathcal{O}(1 / T)$, we can write the following affine relation between the variance of the flicker-removed stack $\widehat{u}(x, y, t)$ and the expected value of $u(x, y, t)$ (which is not affected by the light flickering):

$$
\operatorname{Var}(\widehat{u}(x, y, t))=g E(u(x, y, t))+\sigma^{2}-g \mu,
$$

which is the same relation as that in the standard model (1.3).

Remark 1. In practice, each term of the sum in the definition (3.8) of $v^{*}$ is weighted by $e^{-\left(\bar{u}(x, y)-u^{*}\right)^{2} /\left(2 \sigma_{u^{*}}^{2}\right)}$ and is weighted similarly for $v_{*}$ in the definition (3.14). This acts as a soft threshold in the definition of $\mathcal{S}^{*}$ or $\mathcal{S}_{*}$ as noted in another context in [15]. By construction, $v^{*}(t)$ and $v_{*}(t)$ are nonlocal estimators [8].

Remark 2. Since very small values for $\gamma_{t}$ yield $E\left(v^{*}(t)\right)-1 \simeq E\left(v_{*}(t)\right) \simeq 0$ (cf. (3.9)(3.10) with $\gamma_{t} \simeq 0$ ), the accuracy of the estimated $\mu$ obtained through linear regression should increase with the standard deviation $\sigma_{\gamma}$.

Remark 3. The choice of the $99 \%$ and $1 \%$ quantiles gives satisfactory results in all experiments of section 4 . However, these values can be slightly altered if, in particular images, the cardinality of the set $\mathcal{S}^{*}$ or $\mathcal{S}_{*}$ is not large enough.

3.3. Linear regression. From (3.24), a simple linear regression over the scatter plot of the time sample variance of the $\widehat{u}(x, y, t)$ against the time sample mean of the $u(x, y, t)$ gives us estimations of the slope $g$ and intercept $\sigma^{2}-g \mu$, and hence an estimation of $\sigma$ since $\mu$ is known at this stage by (3.16).

Once $\gamma_{t}$ and $\mu$ have been estimated, another possibility could have been to plug these values into the coefficients of (2.21) estimated through quadratic regression in order to get estimations of $g$ and $\sigma$. We have noted that the obtained estimations were not as good as those obtained by linear regression with (3.24), probably because of the more pronounced effect of PRNU due to the quadratic term and of the correlation between the linear and the quadratic term. We therefore do not use this quadratic regression to estimate the camera parameters. The result of this regression is, however, used to obtain an estimation of the covariance matrix $\operatorname{Cov}(\alpha, \beta)$.

Copyright $\odot$ by SIAM. Unauthorized reproduction of this article is prohibited. 
Input: A series of $T$ raw images $u(x, y, t)$ of a static scene affected by a flickering light and possibly by vibrations.

1. Compute sample expectation $\bar{u}(x, y)$ and sample variance $\bar{\sigma}_{u}^{2}$ at every pixel. (section 2.3).

2. Remove clipped pixels and pixels such that $2 T\|\nabla \bar{u}\|^{2} / \bar{\sigma}_{u}^{2}>9.21$ (section 3.1).

3. For every $t$, compute $v^{*}(t)$ (eq. (3.8)) and $v_{*}(t)$ (eq. (3.14)). A linear regression on the scatter plot $\left(v_{*}(t), v^{*}(t)-1\right)$ gives the slope $K$ (eq. (3.12)), and subsequently estimations of $\mu$ (eq. (3.16)) and of $\gamma_{t}$ for every $1 \leq t \leq T$ (eq. (3.17)), and hence $\sigma_{\gamma}^{2}$.

4. A linear regression between the sample variance of $\widehat{u}(x, y, t)$ (eq. (3.18)) and the sample expectation of $u(x, y, t)$ gives a slope of $g$ and an intercept of $\sigma^{2}-g \mu$ (eq. (3.24)), and hence an estimation of the camera parameters $g$, $\sigma^{2}-g \mu, \sigma$.

Independently, an estimation of the covariance matrix of the vibration process $\left(\alpha_{t}, \beta_{t}\right)$ is obtained from (2.20) by a multiple linear regression between $\bar{\sigma}_{u}^{2}$ and $\bar{u}^{2}, \bar{u},(\partial \bar{u} / \partial x)^{2},(\partial \bar{u} / \partial y)^{2}$, and $\partial \bar{u} / \partial x \cdot \partial \bar{u} / \partial y$ (performed on the whole data set, including pixels affected by vibrations).

Output: An estimation of $g, \mu, \sigma, \sigma^{2}-g \mu, \gamma_{t}, \operatorname{Cov}(\alpha, \beta)$, and $\sigma_{\gamma}$.

Figure 2. Estimation of the camera parameters and of the external parameters by stacking images affected by illumination flickering and vibrations.

3.4. Summary: Algorithm. While simple linear regression permits us to estimate the camera parameters $g, \mu, \sigma$, and $\sigma_{\gamma}^{2}$ as described above, multiple linear regression with (2.20) (not restricted to pixels unaffected by vibrations) additionally gives an estimation of the covariance matrix of the joint process $\left(\alpha_{t}, \beta_{t}\right)$. The whole procedure is summarized in the algorithm of Figure 2.

3.5. Balancing the rolling shutter effect. In any digital single-lens reflex (DSLR) camera with a focal-plane shutter, pixels are not illuminated simultaneously. Such a shutter is indeed made of two curtains running one after the other across the sensor plane. This is all the more noticeable when fast shutter speeds are used, especially with speeds faster than X-sync, defined as the fastest shutter speed at which the whole sensor is completely exposed. This feature, illustrated in Figure 3, gives so-called rolling shutter effects. CMOS cameras with an electronic shutter are also affected since the image is not captured instantaneously but instead row by row. The rolling shutter effect does not change the quadratic relation of (2.21), which only involves the variance $\sigma_{\gamma}^{2}$ and is based on a local argument, but this renders ineffective the image-wide estimation of each $\gamma_{t}$ in section 3.2.

The proposed workaround consists of considering that $\gamma_{t}$ depends on the row $y$ (since the DSLR of section 4, as most DSLRs, has vertical-travel shutters). The expectation of $v^{*}(t)$ defined in (3.8) is now

$$
E\left(v^{*}(t)\right)=\frac{1+\frac{1}{\# \mathcal{S}^{*}} \sum_{(x, y) \in \mathcal{S}^{*}} \gamma_{t}(y)+\mu /\left(u^{*}-\mu\right)}{1+\mu /\left(u^{*}-\mu\right)},
$$

Copyright ( $)$ by SIAM. Unauthorized reproduction of this article is prohibited. 

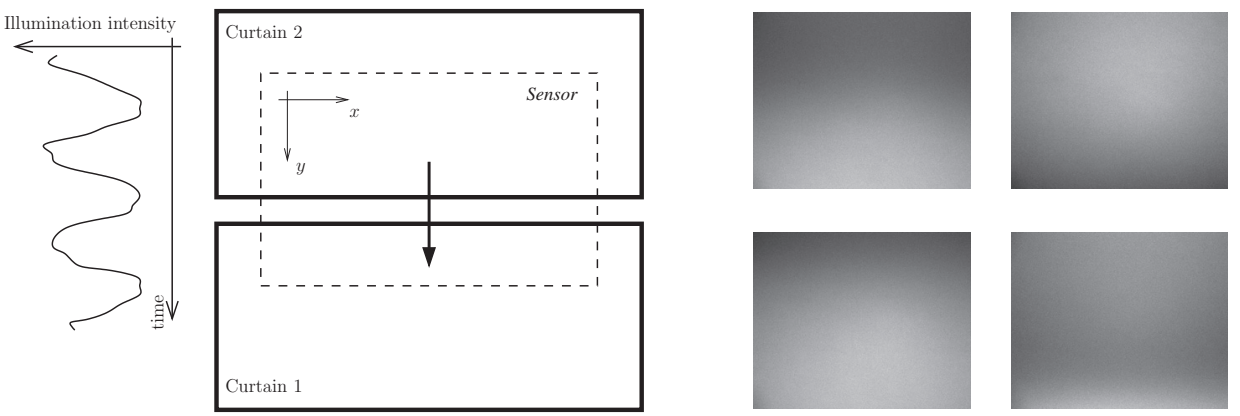

Figure 3. Illustration of the rolling shutter effect. Left: the two curtains go from the top to the bottom, uncovering a part of the sensor. If the scene is illuminated by a flickering source, the illumination intensity varies during the travel of the curtains. Right: four photographs of a blank sheet of paper uniformly illuminated by a neon tube light, under an exposure time of $1 / 1000 \mathrm{sec}$. The horizontal dark strips are caused by the rolling shutter effect (corners are darker because of vignetting). The position of the strips varies from image to image.

and the expectation of $v_{*}(t)$ defined in (3.14) is

$$
E\left(v_{*}(t)\right)=-\left(\frac{1}{\# \mathcal{S}_{*}} \sum_{(x, y) \in \mathcal{S}_{*}} \gamma_{t}(y)\right) \frac{\mu}{u^{*}}\left(u^{*}-u_{*}\right) .
$$

The ratio between $v_{*}(t)$ and $v^{*}(t)-1$ is now

$$
-\frac{\# \mathcal{S}^{*}}{\# \mathcal{S}_{*}} \frac{\sum_{(x, y) \in \mathcal{S}_{*}} \gamma_{t}(y)}{\sum_{(x, y) \in \mathcal{S}^{*}} \gamma_{t}(y)} \mu\left(1-\frac{u_{*}-\mu}{u^{*}-\mu}\right)
$$

instead of $K$ given by (3.15). Generally speaking, since the sets $\mathcal{S}^{*}$ and $\mathcal{S}_{*}$ differ, this quantity is likely to be a biased estimation of $K$, giving a biased estimation of $\mu$. However, if the distribution along $y$ of both sets is alike, then the ratio $\frac{\# \mathcal{S}^{*}}{\# \sum_{*}} \sum_{\mathcal{S}_{*} \gamma_{t}(y)} \gamma_{\mathcal{S}_{t}(y)}$ is close to 1 . Even in the limit case, where $\mathcal{S}^{*}$ and $\mathcal{S}_{*}$ form two clusters with a large illumination difference, the distribution along time $t$ of the introduced bias is likely to be symmetrically distributed around 1, and consequently should be smoothed out by the linear regression over $\left(v^{*}(t)-\right.$ $\left.1, v_{*}(t)\right)$ used to estimate first $K$, then $\mu$. Consequently, even for fast shutter speed, we keep using the image-wide procedure of section 3.2 to estimate $\mu$, which gives good experimental results.

However, we adapt the estimation of $\gamma_{t}$ from section 3.2 and use, instead of (3.8), the modified nonlocal estimator $v^{*}(y, t)$,

$$
v^{*}(y, t)=\frac{1}{N_{y} \# \mathcal{S}^{*}}\left(\sum_{\left(x^{\prime}, y^{\prime}\right) \in \mathcal{S}^{*}} e^{-\left(y^{\prime}-y\right)^{2} /\left(2 \sigma_{y}^{2}\right)} v\left(x^{\prime}, y^{\prime}, t\right)\right),
$$

with a normalization term $N_{y}=\sum_{\left(x^{\prime}, y^{\prime}\right) \in \mathcal{S}^{*}} e^{-\left(y^{\prime}-y\right)^{2} /\left(2 \sigma_{y}^{2}\right)}$. This amounts to estimating $\gamma_{t}(y)$ on horizontal strips of a fixed width centered at row $y$. We take $\sigma_{y}=50$ pixels. Of course, a rigorous setting of the width should involve the flickering frequency, the shutter speed, and the sensor $y$-size (or the $y$-size of a cropped image). We leave this for a future work.

Copyright (c) by SIAM. Unauthorized reproduction of this article is prohibited. 
We obtain an estimation of $\gamma_{t}(y)$ from (3.17), which is then plugged into (3.18), and the same computation as in (3.19)-(3.23) still gives the affine relation (3.24).

4. Experimental results. The aim of this section is to assess the proposed estimation method from a series of images under the same lens aperture, shutter speed, and ISO setting (gain parameter). The algorithm of Figure 2 gives an estimation of the covariance matrix of the vibration process $\left(\alpha_{t}, \beta_{t}\right)$, the relative fluctuation of the light intensity $\gamma_{t}$, and the parameters governing the noise model, namely the gain $g$, the offset $\mu$, and the readout noise $\sigma$. This section proves that satisfactory estimations can be obtained, thanks to illumination flickering. As a sanity check, we make a comparison with the software provided by Foi et al. ${ }^{1}$ (implementing [21] and based on the analysis of a single image, consequently not affected by flickering and vibrations) which gives estimations of $g$ and $\sigma^{2}-g \mu$. In order to compare estimations on the same amount of data, this software is run on a composite image where all $T$ images from the stack have been placed in a $\sqrt{T} \times \sqrt{T}$ mosaic. Its default parameters are used.

The reader should note that the aim of the following experimental benchmark is to compare the values output by the algorithm described in Figure 2 with the values obtained by well established methods, namely [21] or the photon transfer method (for real data). A comparison with multi-image estimation techniques such as [20] or [27] would require a separate study.

Section 4.1 details a running example. Section 4.2 presents results for a synthetic data set. Section 4.3 gives results for actual raw data from a PCO Sensicam QE camera and two Canon EOS DSLR cameras, namely the old EOS 350D and the recent EOS 6D. A comparison with the results of the photon transfer method is also given. For visualization purposes, the contrast of the linear raw images has been modified in the figures. The camera parameters are given in "gray-level" units as in [47].

4.1. Running example. Figure 4 shows an illustrative example. $T=100$ images of an $\mathrm{X}$-Rite Colorchecker illuminated by a neon tube light are taken with a Canon EOS 6D camera (row "ISO 6400" in Table 5, hence a fast shutter speed of 1/320 sec, prone to the rolling shutter effect). The scatter plot of $v_{*}(t)$ against $v^{*}(t)-1$ shows the proportionality relation of slope $K<0$. In this case, the assumptions of section 3.5 are satisfied, and the proportionality relation is nearly perfect in spite of the rolling shutter effect. The light flickering $1+\gamma_{t}(y)$ is estimated with the method of section 3.5 and $\sigma_{\gamma}=0.24$ is found. The dependence of $\gamma_{t}(y)$ on $y$ is noticeable. The graph of the sample variance of $u(x, y, t)$ against the sample expectation has a quadratic trend as predicted by (2.21). The plot for $\widehat{u}(x, y, t)$ is not linear when $\gamma_{t}$ is assumed constant over the image: pixels belonging to some color patches are not adequately corrected, which explains the clusters out of the linear trend shown on the upper part of the bottom left graph. In this case, the regression line does not give a slope and an intercept consistent with the expected gain, offset, and readout noise. With an estimation performed as in section 3.5, the trend becomes linear and a correct estimation of the camera parameters is possible (see section 4.3). The green points are outliers removed by the first step of the algorithm (pixels affected by vibrations; see section 3.1), and the estimations of the camera parameters are based on the blue points.

\footnotetext{
${ }^{1}$ Version 2.31, available at http://www.cs.tut.fi/ foi/sensornoise.html.
} 

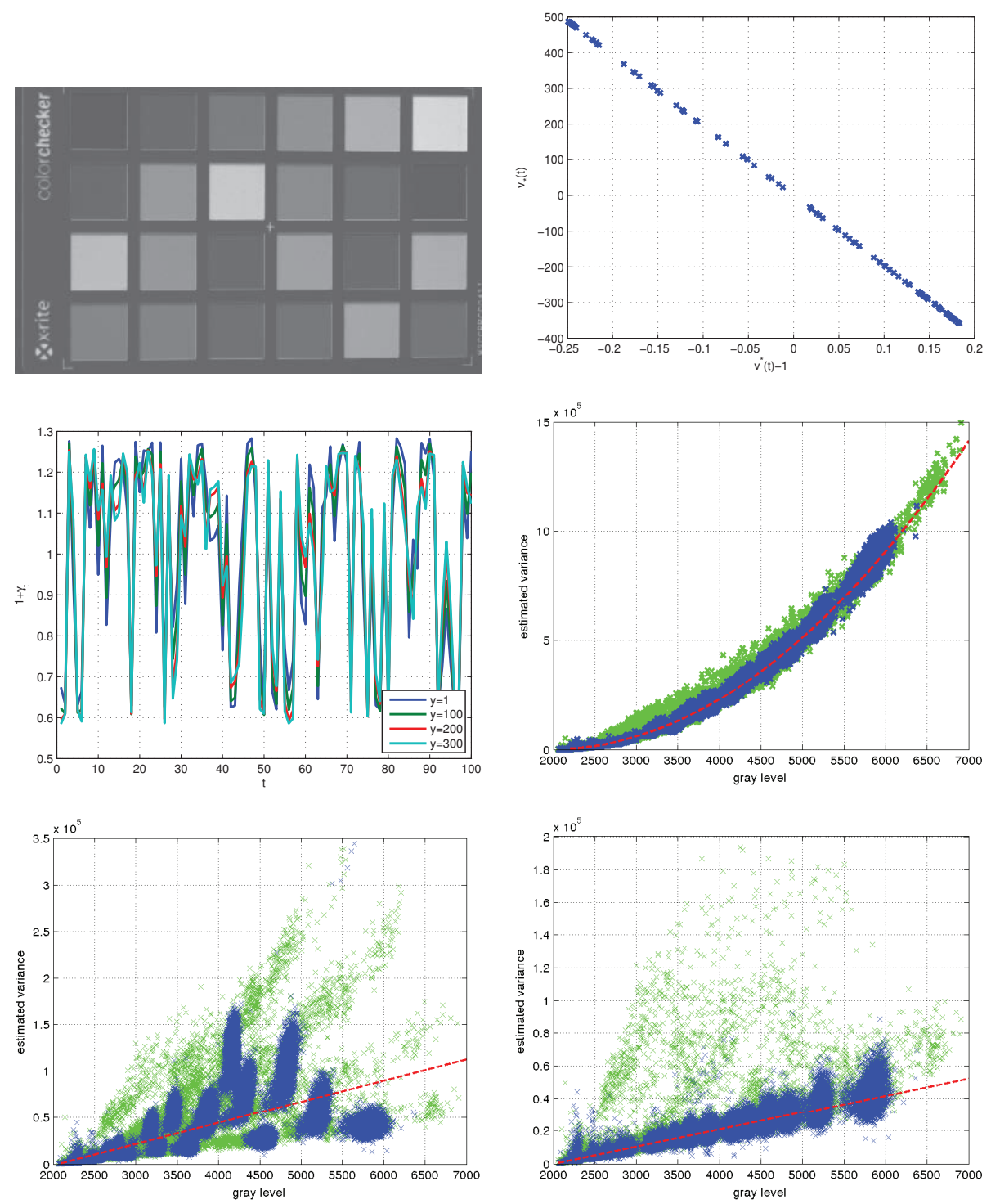

Figure 4. Running example. Top: an image from the series, and the graph of $v_{*}(t)$ against $\left.v^{*}(t)-1\right)$. Middle: estimation of the relative fluctuation of the light $1+\gamma_{t}(y)$ for several values of $y$, and graph of the sample variance of $u(x, y, t)$ against the sample mean of $u(x, y, t)$. Bottom: graph of the sample variance of $\widehat{u}(x, y, t)$ against the sample mean of $u(x, y, t)$ (image-wide estimation of $\gamma_{t}$ ), and the same statistics when taking into account the rolling shutter effect.

4.2. Synthetic data. In this experiment, stacks of images are generated from reference images using the model of (2.1). More precisely, $p(x, y)$ is the intensity value of a $512 \times 512$ reference image scaled to $[0, A]$, where $A>0$ is the maximum number of generated electrons at a given photosite. For every $t, \alpha_{t}, \beta_{t}$, and $\gamma_{t}$ are independent random numbers simulated following a Gaussian distribution of standard deviation equal to, respectively, $\sigma_{\alpha}, \sigma_{\beta}$, and $\sigma_{\gamma}$. The value of $p\left(x+\alpha_{t}, y+\beta_{t}\right)$ is obtained by linear interpolation of the scaled reference

Copyright (c) by SIAM. Unauthorized reproduction of this article is prohibited. 
image $p$ (a Gaussian blur of a standard deviation of 0.5 pixels is first applied to the reference images to simulate optical blur). A stack $u(x, y, t)$ of $T$ images is randomly generated from the Poisson-Gaussian model of (2.1) and quantized over 12 bits afterward. Note that quantization irremediably leads to loss of information. It should also be noted that the intensity $p(x, y)$ may come from a noisy reference image. This feature does not play any role in our estimation process, since this noisy component is static and does not change along the stack. This may, however, give an additional bias in the estimation by the software implementation of [21].

Since the results vary between realizations of the stochastic processes, 50 such simulations are run, which permits us to estimate mean values together with their standard deviations. In the remainder of this section we give the mean \pm twice the standard deviation obtained in this Monte Carlo approach.

Three camera models and three external conditions are tested, which gives nine different experimental settings for a given reference image. The corresponding parameter values are listed in Table 1. Synthetic cameras A-C have typical noise levels for varying ISO values of a 12-bit camera. Parameter $A$ is set so that the quantized intensity values span the 12-bit range. In Table 1, the ranges of vibration and the flickering amplitudes correspond to typical observations. For example, the vibration amplitude in external conditions 3 corresponds to the experimental measurements in [53]. External conditions 1 are very demanding, with vibrations whose amplitudes are likely to be as large as one pixel, and with large illumination changes between successive images.

Six reference images are tested; see Figure 5 . The first is a synthetic calibration target with large homogeneous areas (Synthetic). The others come from the USC-SIPI Image Database. ${ }^{2}$ As we shall see, the Synthetic image is well adapted to the software of Foi et al. Nevertheless, our method also needs iso-value sets for the nonlocal estimation of section 3.2 (cf. the $99 \%$ quantile level set in (3.8) and the 1\% quantile level set in (3.14)), and the images should be smooth enough so that pixels are not overly removed because of large gradients. The remaining reference images are chosen to test the influence of irregular textures. Such textures not only make it difficult to segment the image, but also give a larger proportion of pixels affected by the vibrations (for instance, $59.1 \%$ for Boat against $8.8 \%$ for Synthetic, in the sense of section 3.1).

A first experiment consists in assessing the influence of the number $T$ of stacked images. Figure 6 shows the typical evolution of the relative precision of the estimated parameters together with their $95 \%$ confidence intervals ( \pm twice the standard deviation of the estimation, shown as colored plots in the figure). The reference image is the Synthetic target. Here "relative precision" means that the values of interest are divided by the ground truth value. The estimated confidence intervals show a $1 / \sqrt{T}$ decrease as in every Monte Carlo approach. This is, however, limited by the quantization of the data which imposes a lower bound on the accuracy (see, e.g., [56]). From these results, the parameter $T$ is set to 100 in the remainder of the paper. As we can see, a smaller number of stacked images would also give acceptable results.

Tables 2 and 3 give comprehensive results for the Synthetic reference image and for the Boat image, respectively. The first row in each table gives the experiment number. Experi-

\footnotetext{
${ }^{2}$ available at http://sipi.usc.edu/database/.
} 


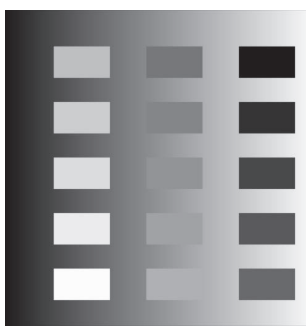

1. Synthetic.

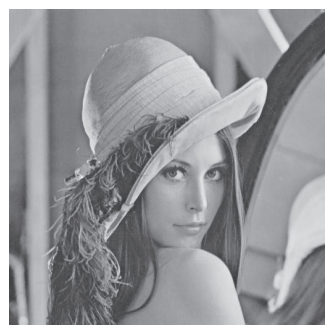

4. Lena.

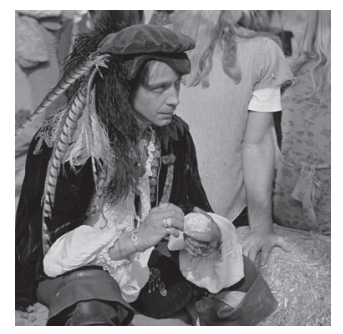

2. Man

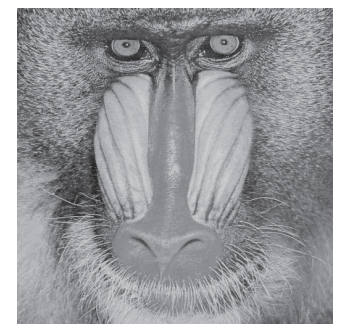

5. Mandrill.

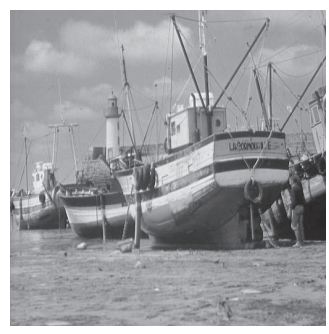

3. Boat.

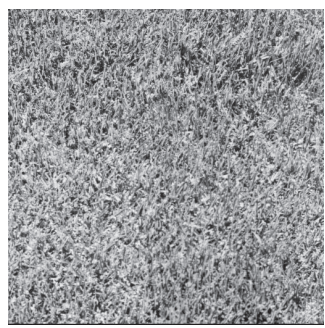

6. Grass.

Figure 5. Reference images, with an increasing high-frequency texture content. These images have a size of $512 \times 512$ pixels, except for Man which has a size of $1024 \times 1024$ pixels.

Table 1

Parameter values: camera settings on the left; experimental conditions on the right.

\begin{tabular}{|c||c|c|c|}
\hline Camera & A & B & C \\
\hline$A$ & 600 & 3,000 & 10,000 \\
\hline$g$ & 5 & 1 & 0.3 \\
\hline$\mu$ & 200 & 100 & 40 \\
\hline$\sigma$ & 15 & 8 & 3 \\
\hline
\end{tabular}

\begin{tabular}{|c||c|c|c|}
\hline Exp. & 1 & 2 & 3 \\
\hline$\sigma_{\gamma}$ & 0.1 & 0.01 & 0.05 \\
\hline$\sigma_{\alpha}$ & 0.3 & 0.1 & 0.01 \\
\hline$\sigma_{\beta}$ & 0.2 & 0.05 & 0.02 \\
\hline
\end{tabular}

ment "X n" deals with the camera "X" in the experimental conditions " $\mathrm{n}$ " in Table 1. For each of the estimated parameters (in columns 3-9), the ground truth (GT) is given, as well as the result of the proposed algorithm (estim.), and for $g$ and $\sigma^{2}-g \mu$ the output of the software by Foi et al. is shown.

We can see that with the Synthetic reference image, all parameters are very accurately estimated and the actual values are almost always in the confidence interval of the estimation. Large vibrations (conditions 1 ) give less accurate estimations of the slope $\sigma^{2}-g \mu$ and consequently of $\sigma$. The results of the software provided by Foi et al. are comparable, and, of course, are not affected by flickering or vibrations. This experiment proves that, with a simple synthetic image stack, the proposed approach allows us to accurately estimate not only the camera parameters $g, \mu, \sigma$ but also the external parameters of the experimental setting, namely $\sigma_{\gamma}, \sigma_{\alpha}$, and $\sigma_{\beta}$, benefitting from illumination flickering.

The textured Boat reference image is more challenging. On the one hand, we can see that, in this case, the performance of the method by Foi et al. deteriorates. For some textured reference image, its results are even totally wrong. It should be noted that a robust version of their algorithm has been recently proposed [4]. On the other hand, the proposed algorithm

Copyright (c) by SIAM. Unauthorized reproduction of this article is prohibited. 

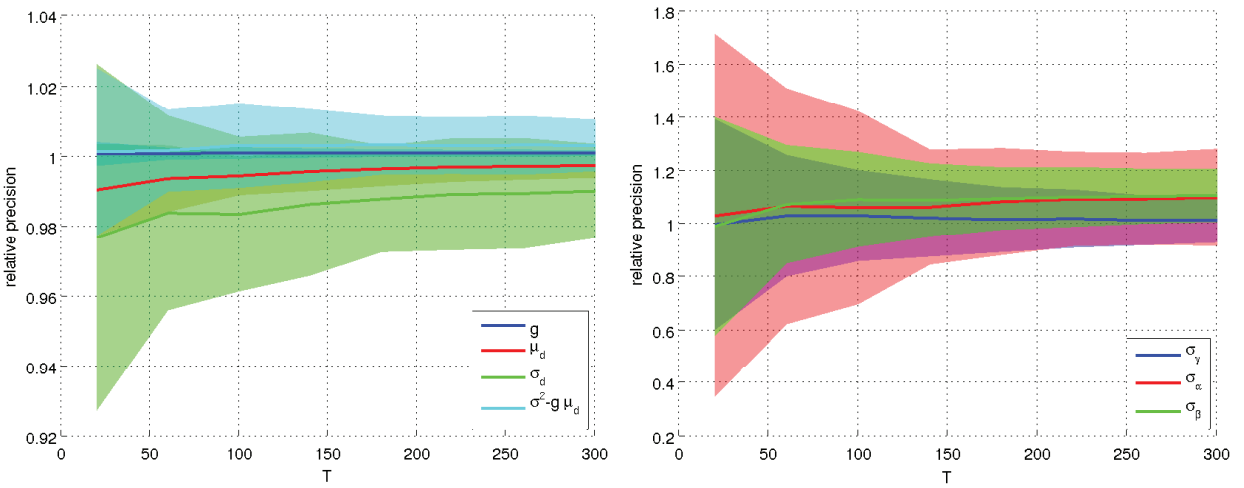

Figure 6. Evolution of the relative precision of the estimated parameters with respect to the number of stacked images (left: intrinsic parameters $g, \mu, \sigma, \sigma^{2}-g \mu$; right: external parameters $\sigma_{\gamma}, \sigma_{\alpha}, \sigma_{\beta}$ ). Camera A is used with conditions 3 and the Synthetic target as reference image.

gives quite reliable estimations of $g$ (the actual value is within the estimated confidence interval, which is quite small). The variability of the estimated $\mu$ is very large in experimental conditions 2 (with $\sigma_{\gamma}=0.01$ ), as expected from Remark 2 in section 3.2. Concerning the estimation of $\sigma^{2}-g \mu$ (and consequently of $\sigma$ ) the vibrations strongly affect the accuracy and the variability. However, a reliable estimation is still obtained in experimental conditions 3 (moderate vibrations). Note that in all of the experimental conditions, the estimation of the light variability $\sigma_{\gamma}$ is very good, and the estimations of the variabilities $\sigma_{\alpha}$ and $\sigma_{\beta}$ of the vibrations are quite accurate, even with strong vibrations (experimental conditions 1 and 2 ).

Figures 7 and 8 show the error bars (95\% confidence intervals) for the estimation of the intrinsic parameters $g, \mu, \sigma^{2}-g \mu$, and $\sigma$, and of the extrinsic parameters $\sigma_{\gamma}, \sigma_{\alpha}$, and $\sigma_{\beta}$, respectively. Concerning the intrinsic parameters in Figure 7 , we can see that the estimation of the gain deteriorates in the case of high-frequency textures (reference images 5 and 6), especially for large vibrations (experimental conditions 1 ). In other cases, the relative error is well below $10 \%$. As predicted by the theory, the quality of the estimation of $\mu$ is poor for low $\sigma_{\gamma}$ (conditions 2), except for lightly textured images (reference images 1 and 2). The relative error is larger when $\mu$ decreases. The estimation of the slope $\sigma^{2}-g \mu$ also deteriorates on textured images, and it is all the more noticeable as the vibration amplitude is large (conditions 1). With tiny vibrations (conditions 3), corresponding to more realistic cases, a reliable estimation is available in all cases, although the variability is large for textured images. The estimation of $\sigma$ directly follows the estimation of $\sigma^{2}-g \mu$. Concerning the extrinsic parameters in Figure 8, we can see that the estimation of the flicker amplitude $\sigma_{\gamma}$ is reliable in spite of a deterioration with textured images (reference image 6 ) in the case of small $\sigma_{\gamma}$ (conditions 2), and that the estimations of the vibration amplitudes $\sigma_{\alpha}$ and $\sigma_{\beta}$ are slightly overvalued, with unreliable estimation of extreme amplitudes ( $\sigma_{\alpha}$ in conditions 1 ) with the high gain camera $\mathrm{A}$ in the untextured reference image 1).

Copyright ( ) by SIAM. Unauthorized reproduction of this article is prohibited. 


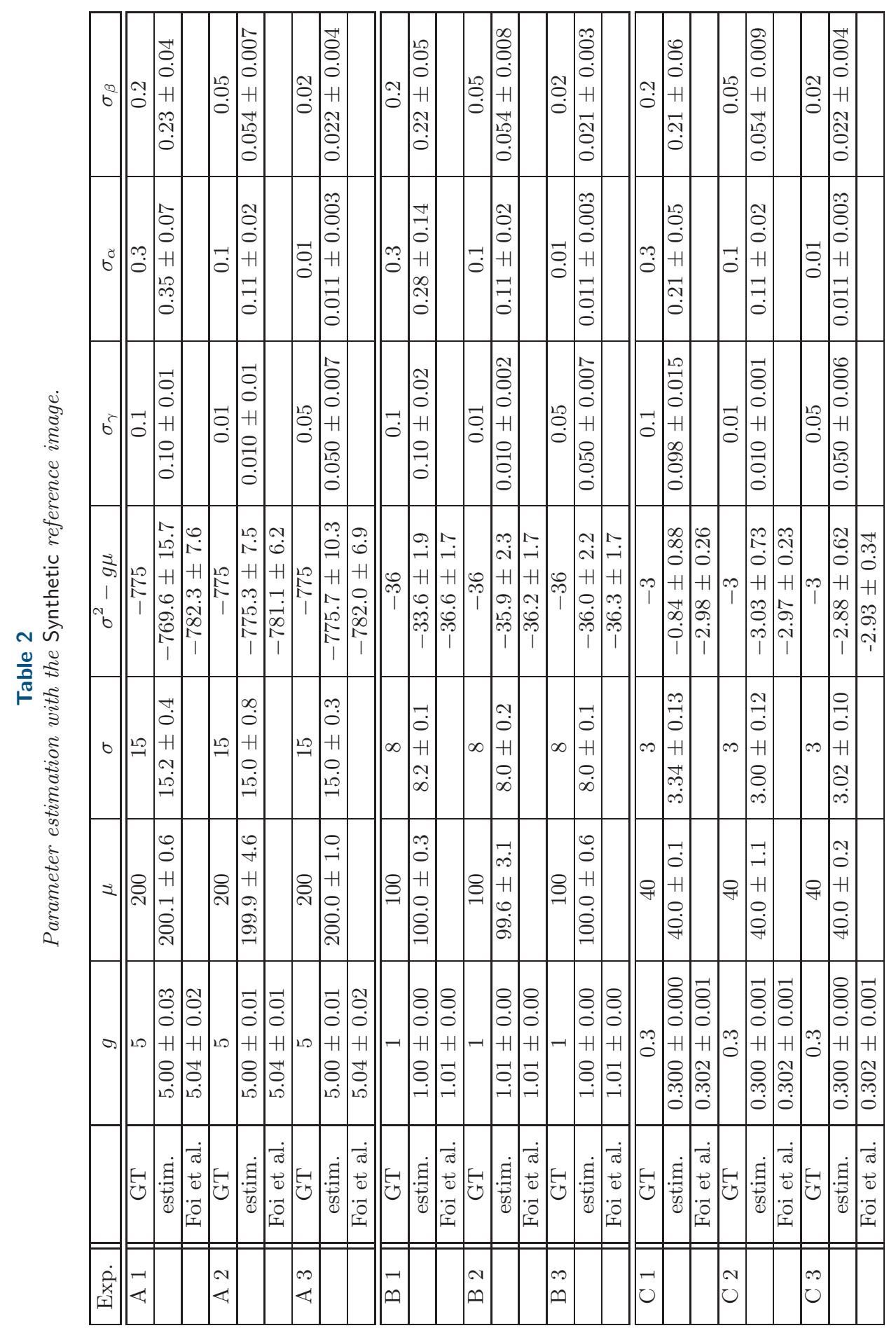




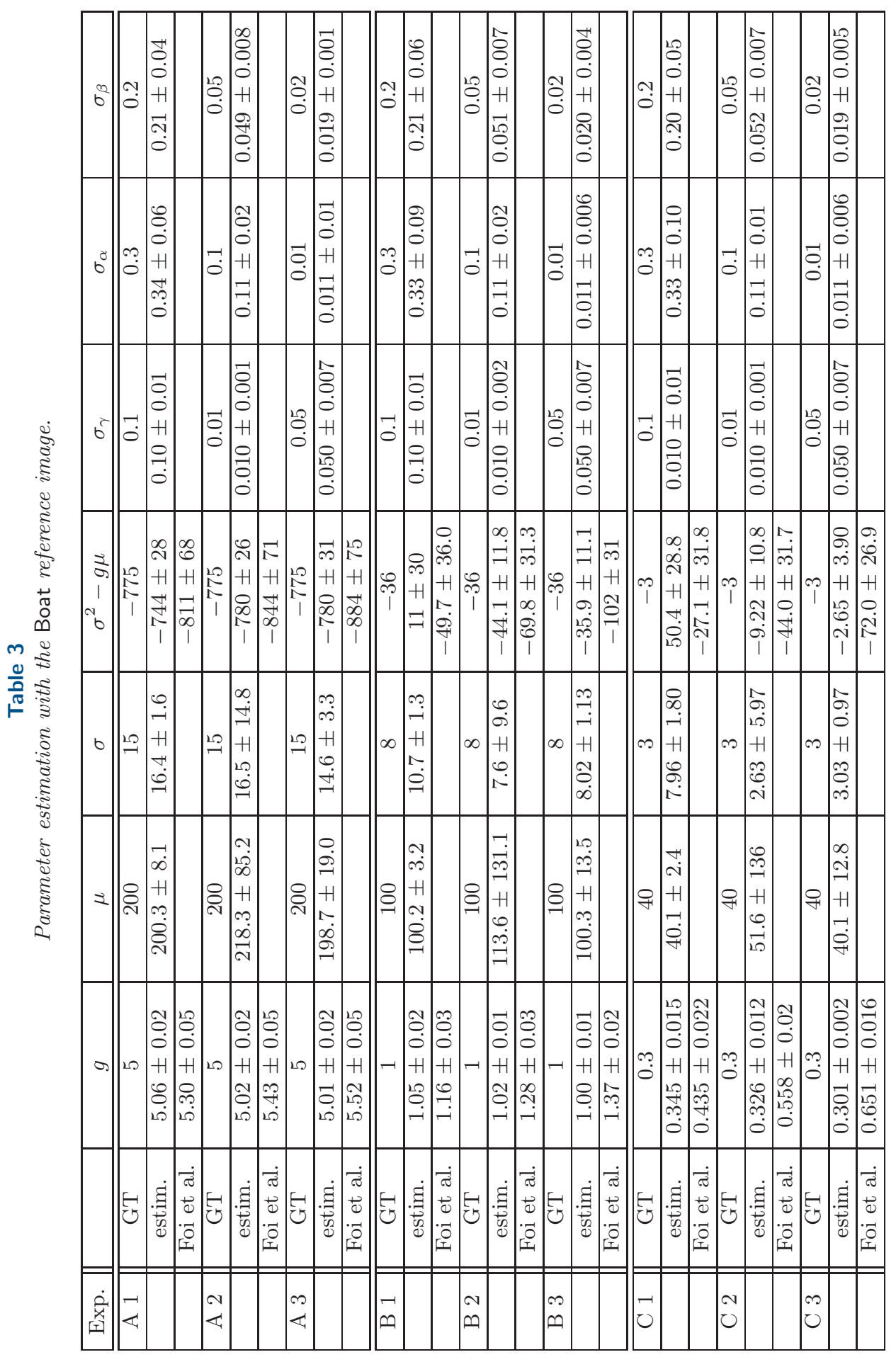



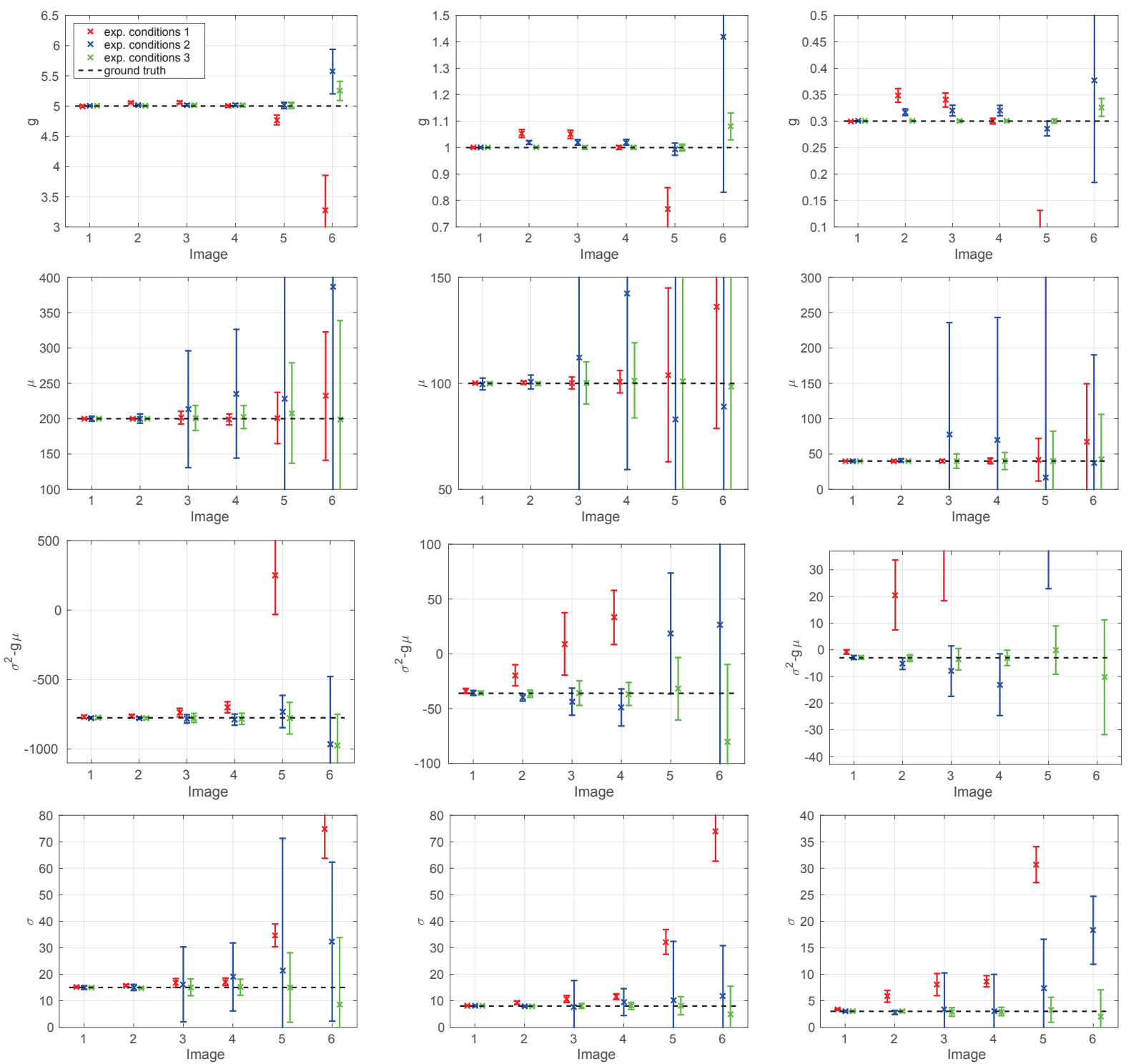

Figure 7. Estimation of the intrinsic camera parameters $\left(g, \mu, \sigma^{2}-g \mu\right.$, and $\sigma$, from top to bottom) for camera A (left), camera B (middle), camera C (right), for reference images 1-10. Information in the inset of the top left plot holds for all images. 

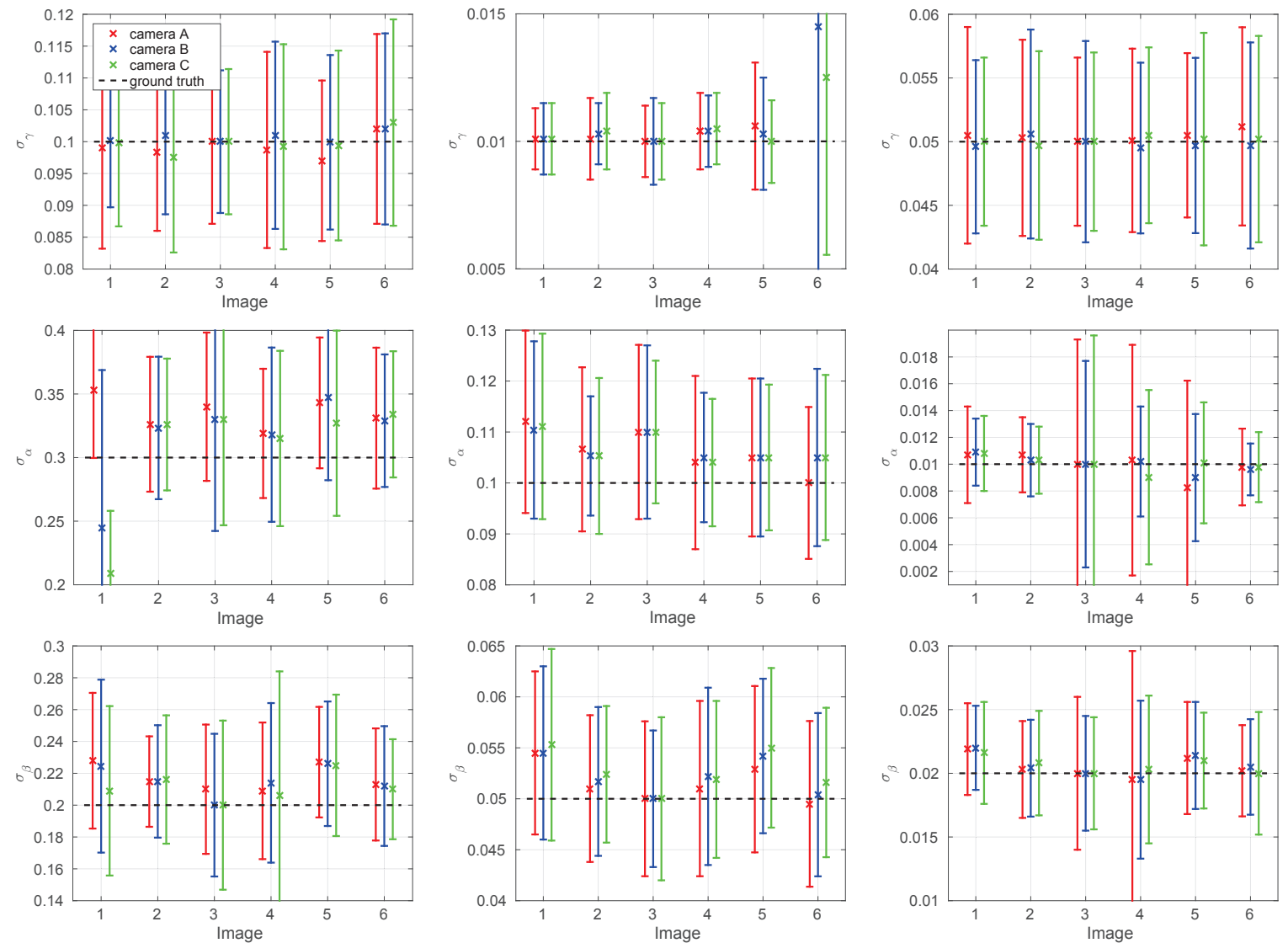

Figure 8. Estimation of the extrinsic parameters $\left(\sigma_{\gamma}, \sigma_{\alpha}, \sigma_{\beta}\right.$, from top to bottom) for conditions 1 (left), conditions 2 (middle), conditions 3 (right) for reference images 1-10. Information in the inset of the top left plot holds for all images.

Copyright ( ) by SIAM. Unauthorized reproduction of this article is prohibited. 
4.3. Real data. In this section, image series from a PCO Sensicam QE and two Canon DSLR cameras are tested.

4.3.1. PCO Sensicam QE. Experiments are performed with a PCO Sensicam QE camera which gives $1040 \times 1376$ 12-bit images. The gain parameter is set to "high." This camera is cooled to $-12^{\circ} \mathrm{C}$, and it has been shown that, for standard exposure times, the noise level is nearly constant (see, e.g., [30, pp. 24-27], where it is shown that this is the case for exposures below $100 \mathrm{~ms}$ ). Values for $g, \mu$, and $\sigma$ are provided by the manufacturer [46], supposedly following the EMVA procedure [47]. We have obtained close values with an independent method on pseudoperiodic grid images [50]; see Table 4.

A series of 100 images of a composite specimen (elongated chips are pieces of sunflower stem and light aggregates are pieces of pith [48], resulting in highly textured images) is taken under a neon light. The exposure time of each image is $13 \mathrm{~ms}$, and the image acquisition is triggered electronically, which gives a constant frequency. The estimation of the noise parameters is given in Table 4. The illumination flickering standard deviation is estimated to $\sigma_{\gamma}=0.044$. This experiment is an example of textured images, which cause the algorithm of [21] to fail. The experiment is illustrated in Figure 9.

The series of Figure 10 highlights a phenomenon to which the proposed method is sensitive. An explanation for the graph of the variance of $\widehat{u}$ against the expectation of $u$ seems to be that, in this scene, a small area is illuminated by a neon light that is different than that illuminating the rest of the scene. The two lights have the same fluctuation amplitude (hence it cannot be noticed on the plot of the variance against the expected intensity), but they apparently show out-of-phase fluctuations (hence the pixels from the small area are not adequately corrected, giving the blue points out of the main linear trend). Here, there are a few such pixels, and hence they do not prevent us from reliably estimating the slope and intercept with linear regression. In this experiment we estimate $\sigma_{\gamma}=0.037$.

Table 4

Noise parameter estimation for a Sensicam QE camera.

\begin{tabular}{|c|c|c|c|c|c|}
\cline { 3 - 6 } \multicolumn{1}{c|}{} & $g$ & $\sigma$ & $\mu$ & $\sigma^{2}-g \mu$ \\
\cline { 2 - 6 } \multicolumn{1}{c|}{} & Manufacturer [46] & 0.519 & 1.15 & 45.46 & -22.26 \\
Figure 9 & {$[50]$} & 0.528 & & & -21.01 \\
\hline Figure 10 & {$[21]$} & 2.06 & & & -307.2 \\
& Proposed method & 0.569 & 2.65 & 63.91 & -29.37 \\
\hline & Proposed method & 0.543 & & & -22.80 \\
& 0.559 & 3.14 & 65.3 & -26.63 \\
\hline
\end{tabular}

With the exposure time being equal to $13 \mathrm{~ms}$, the flickering illumination is integrated and gives a quite low $\sigma_{\gamma}$. Consequently, the accuracy of the estimation of $\mu$, and hence $\sigma$, is moderate (see Remark 2 in section 3.2). Additionally, a quite strong DSNU due to the cooling device is noticed in [30]; this may explain a part of the variability of the measurements. Since the acquisition frequency is constant, light flickering yields a periodic $\gamma(t)$ due to the neon light powered by the $50 \mathrm{~Hz} \mathrm{AC}$ power. We can see that the $v_{*}(t)$ against $v^{*}(t)$ plot is scattered. Before flickering compensation, the variance against intensity expectation plot shows a quadratic trend, as predicted by (2.20)-(2.21). It changes to the affine relation with the corrected variance shown in (3.24). Points whose variance can mainly be explained by

Copyright (c) by SIAM. Unauthorized reproduction of this article is prohibited. 

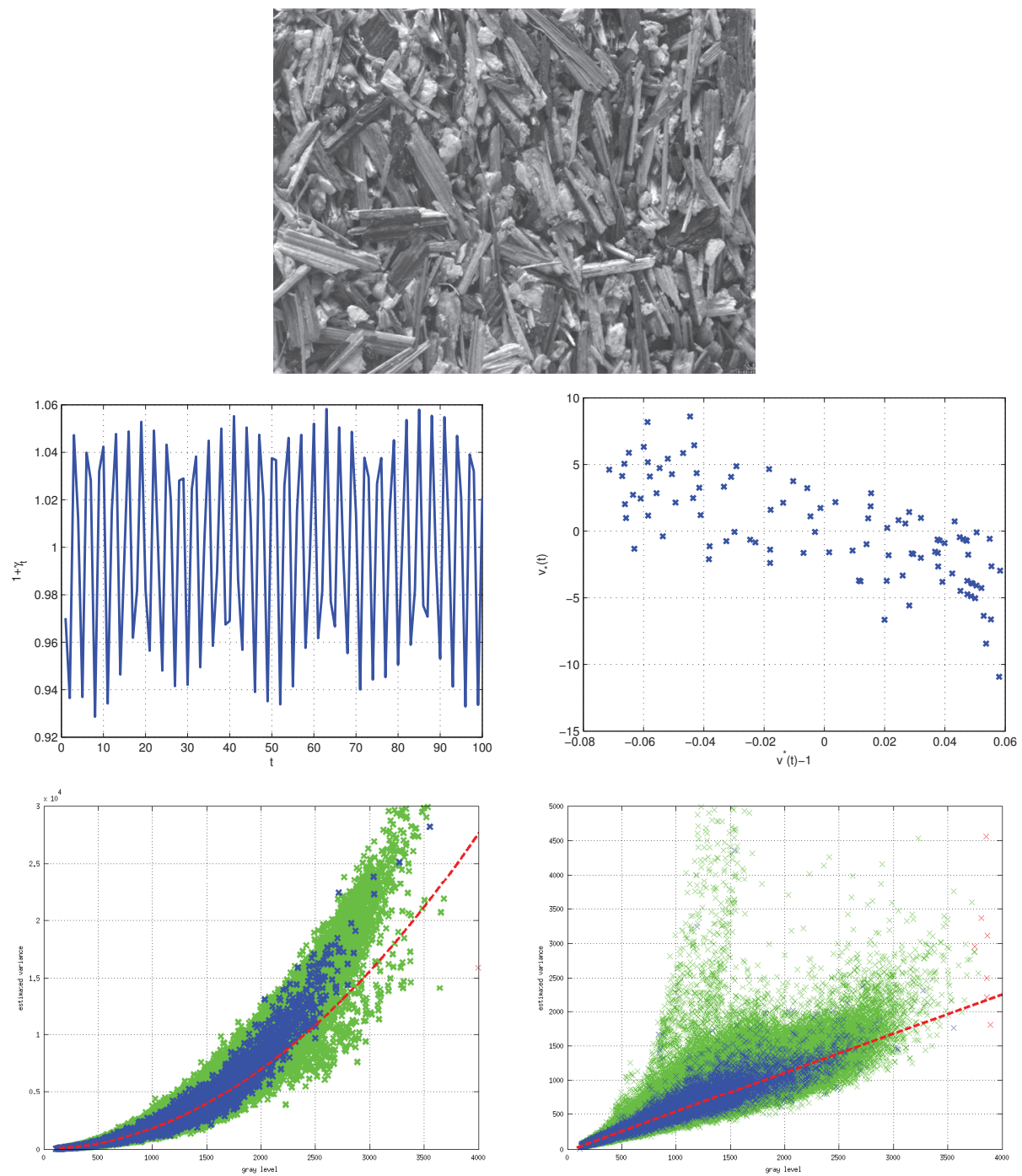

Figure 9. Sensicam QE. From top to bottom and left to right: an image from the series; estimation of the relative fluctuation of the light $1+\gamma_{t}$; graph of $v_{*}(t)$ against $v^{*}(t)-1$; graph of the sample variance of $u(x, y, t)$ against the sample mean of $u(x, y, t)$; graph of the sample variance of $\widehat{u}(x, y, t)$ against the sample mean of $u(x, y, t)$.

vibrations (green points) are of course not adequately corrected, and hence move away from the linear trend.

4.3.2. Canon EOS 350D and EOS 6D cameras. Two Canon DSLR cameras (the 12-bit EOS 350D with an APS-C sensor and the 14-bit EOS 6D with a full-frame sensor) shoot two different scenes illuminated by a neon light: an X-Rite Colorchecker and a cluttered scene (textured objects lying on a desk) as illustrated in Figure 11. Several ISO values are tested and the corresponding exposure times $t$ (depending on the aperture) are given. A series of 100 raw

Copyright (C) by SIAM. Unauthorized reproduction of this article is prohibited. 

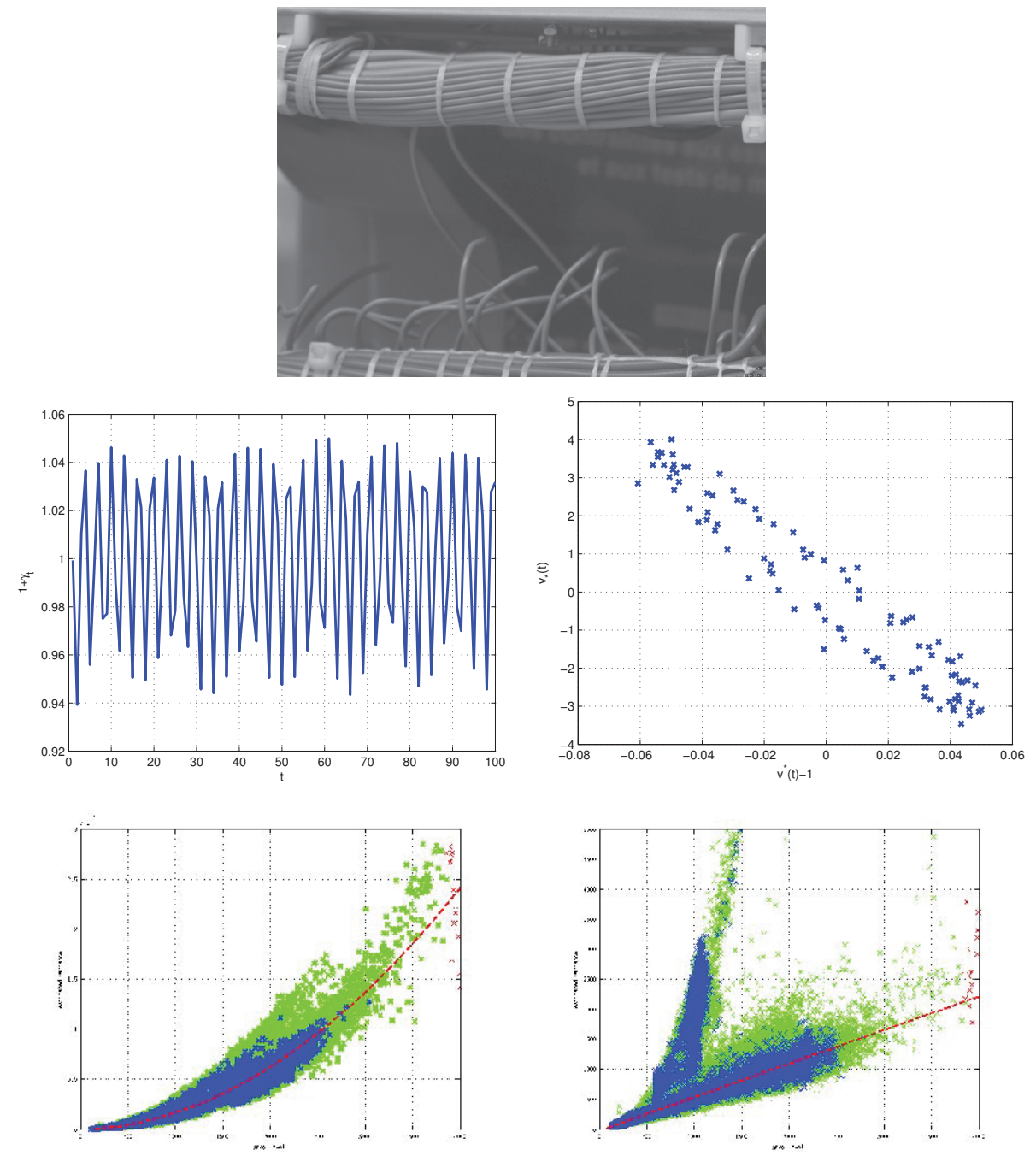

Figure 10. Sensicam QE. From top to bottom and left to right: an image from the series; estimation of the relative fluctuation of the light $1+\gamma_{t}$; graph of $v_{*}(t)$ against $v^{*}(t)-1$; graph of the sample variance of $u(x, y, t)$ against the sample mean of $u(x, y, t)$; graph of the sample variance of $\widehat{u}(x, y, t)$ against the sample mean of $u(x, y, t)$.

images is linearly processed with dcraw, ${ }^{3}$ and the Bayer color channel corresponding to Red is extracted. Other channels give similar results, see [52]. In Table 5, we give the following:

1. The values obtained by the photon transfer method ${ }^{4}$ (the offset $\mu$ is estimated independently over a short exposure dark frame image);

2. the output $g$ and $\sigma^{2}-g \mu$ of the software by Foi et al.;

3. the results of the proposed method;

\footnotetext{
${ }^{3}$ Available at http://www.cybercom.net/ dcoffin/dcraw/. Options: dcraw -c -D -4 -r 1111.

${ }^{4}$ EOS 350D: available at http://www.astrosurf.com/comolli/strum41.htm EOS 6D: available at http://www.astrosurf.com/comolli/strum54.htm
} 
4. the estimation of the slope $g$ and the intercept $\sigma^{2}-g \mu$ of a regression line from $(E(u), \operatorname{Var}(u))$, disregarding the quadratic trend.

ISO settings marked with an asterisk are processed with the method of section 3.5 to deal with the rolling shutter effect.

Some results of the method of [21] suggest that the default software parameters might not be adequate in some situations. The overestimation of $g$ by the fourth method (which does not take the quadratic trend into account) shows that it is important to correct flickering, especially for high ISO values giving fast shutter speeds. We can see that the proposed method correctly estimates not only $g$, but also $\mu$ and, most of the time, $\sigma$.

Table 6 shows the estimations of the vibration parameters and of the standard deviation of the flickering process for the Canon EOS350D with the cluttered scene (other cases are similar; cf. [52]). Concerning the exposure time, since the neon light is powered by $50 \mathrm{~Hz}$ current, its flickering should not be noticeable for low ISO values because of photon integration over an exposure time lower than several tenths of a second. However, $\sigma_{\gamma}$ is estimated at less than 0.01 for $t \simeq 1 / 10 \mathrm{~s}$ and $\sigma_{\gamma} \simeq 0.15$ for $t \simeq 1 / 200 \mathrm{~s}$. This confirms that, here, the light flickering has a noticeable effect, mainly for high ISO. In addition, it shows that a slight variability of the actual exposure, possibly due to mechanical or electronic imperfections, can be measured. This is confirmed by additional experiments under natural light (not shown here; see [52]). It should be noted that, as we can expect, similar exposure times give similar flickering intensity. Except for some situations, the flickering intensity seems to be smaller in the green channels than in the red and blue channels. This might be explained by the sensitivity of these channels to the neon light spectrum. Furthermore, the small values of $\sigma_{\gamma}$ explain the poorer estimation of $\mu$ for low ISO, and hence of $\sigma$. Moreover, the increasing $\sigma_{\gamma}$ makes the bias in the affine method increase with ISO.

The results in Table 6 also show that the proposed estimation of the vibration parameters is quite reliable, since they are within the same order of magnitude for the four channels from the same series. Comparing with Table 5, we can see that the effect of the vibrations tends to vanish when the exposure time increases because of the longer integration time.

5. Conclusion. This paper discussed the effect of illumination flickering (or slightly varying exposure times) and of microvibrations on image noise measurements from a series of images of a static scene, within a Cox-Gaussian model. Image stacking permitted us to easily obtain sample means and sample variances without any prior segmentation step. In the presence of illumination flickering, a quadratic relation between the variance and the expected intensity was established, instead of the standard affine relation. A practical algorithm was proposed, robust to the rolling shutter effect and to the vibrations. Taking advantage of the illumination flickering, it was possible to estimate the gain, the offset, and the readout noise. A large flickering amplitude ensures a higher estimation accuracy, giving results within the range of the photon transfer method. Such an approach may be useful for multi-image denoising methods (as in $[9,34]$ ) or for noise parameter estimation in fluorescence imaging affected by photobleaching (as in [27]), where a nonconstant illumination has been noticed.

Acknowledgments. The authors thank the reviewers for their valuable comments and suggestions. Prof. A. Foi is gratefully acknowledged for making his software publicly available. 


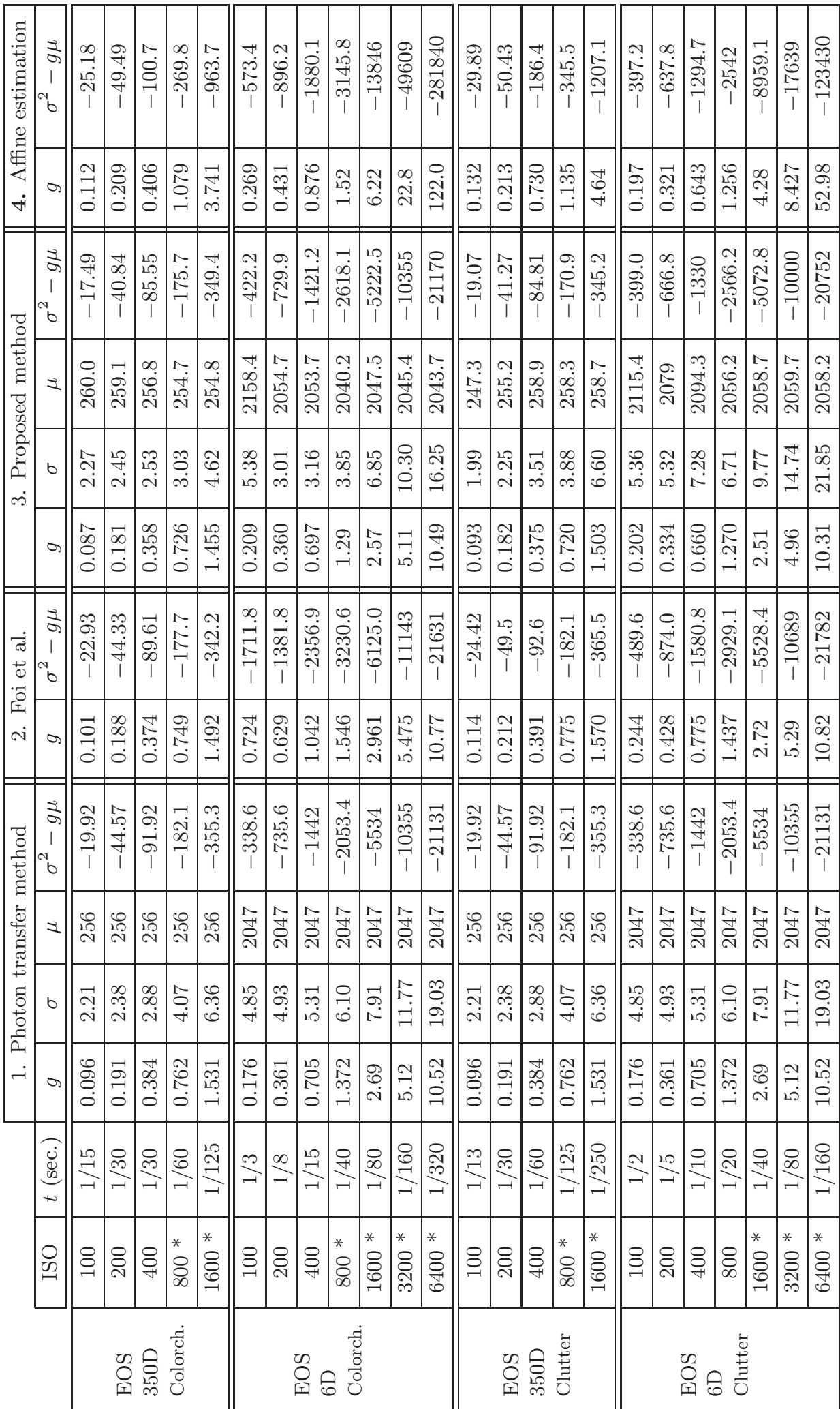



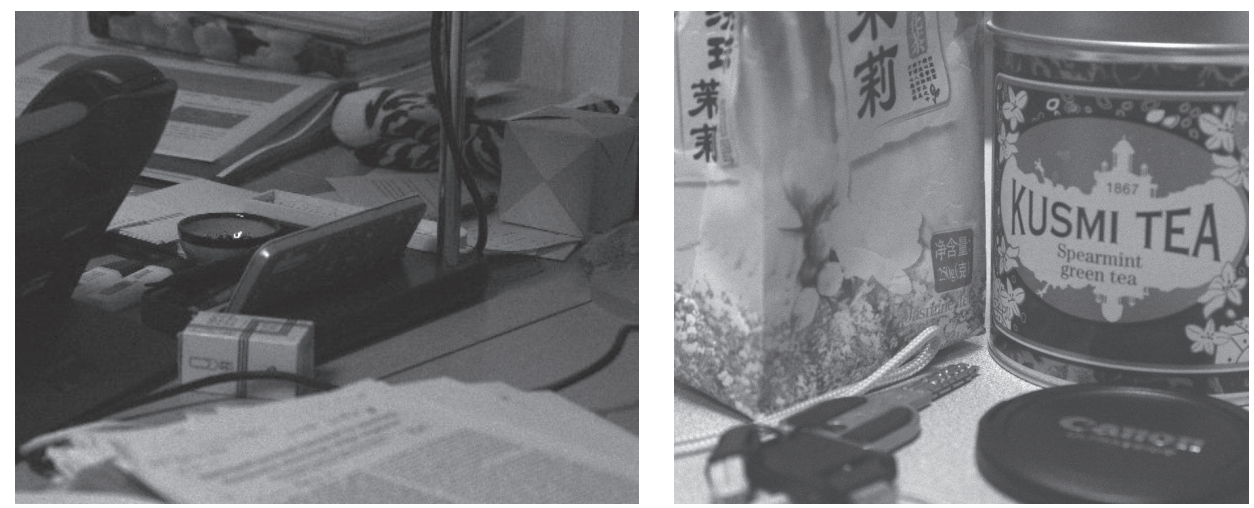

Figure 11. Left: clutter scene for the Canon EOS 350D experiment. Right: clutter scene for the Canon EOS $6 D$ experiment.

Table 6

Estimating vibrations and illumination flickering.

\begin{tabular}{|c|c||c|c|c|c|}
\hline ISO & Channel & $\sigma_{\alpha}$ & $\sigma_{\beta}$ & $\mathrm{Cov}_{\alpha, \beta}$ & $\sigma_{\gamma}$ \\
\hline 100 & $\mathrm{R}$ & 0.047 & 0.19 & -0.0086 & 0.0088 \\
& $\mathrm{G} 1$ & 0.039 & 0.16 & -0.0065 & 0.0088 \\
& $\mathrm{G} 2$ & 0.039 & 0.16 & -0.0064 & 0.0086 \\
& $\mathrm{~B}$ & 0.047 & 0.19 & -0.0086 & 0.0099 \\
\hline 200 & $\mathrm{R}$ & 0.043 & 0.059 & -0.0016 & 0.012 \\
& $\mathrm{G} 1$ & 0.039 & 0.057 & -0.0014 & 0.0092 \\
& $\mathrm{G} 2$ & 0.038 & 0.056 & -0.0013 & 0.0099 \\
& $\mathrm{~B}$ & 0.037 & 0.059 & -0.0014 & 0.012 \\
\hline 400 & $\mathrm{R}$ & 0.037 & 0.049 & -0.0014 & 0.057 \\
& $\mathrm{G} 1$ & 0.028 & 0.045 & -0.0011 & 0.042 \\
& $\mathrm{G} 2$ & 0.025 & 0.045 & -0.00099 & 0.042 \\
& $\mathrm{~B}$ & 0.031 & 0.044 & -0.0012 & 0.059 \\
\hline 800 & $\mathrm{R}$ & 0.042 & 0.078 & -0.0015 & 0.058 \\
& $\mathrm{G} 1$ & 0.03 & 0.073 & -0.001 & 0.042 \\
& $\mathrm{G} 2$ & 0.026 & 0.074 & -0.00083 & 0.041 \\
& $\mathrm{~B}$ & 0.035 & 0.071 & -0.0011 & 0.056 \\
\hline 1600 & $\mathrm{R}$ & 0.066 & 0.043 & -0.0021 & 0.17 \\
& $\mathrm{G} 1$ & 0.027 & 0.032 & -0.00035 & 0.12 \\
& $\mathrm{G} 2$ & 0.027 & 0.03 & -0.00013 & 0.12 \\
& $\mathrm{~B}$ & 0.037 & 0.058 & -0.00032 & 0.17 \\
\hline
\end{tabular}

\section{REFERENCES}

[1] A Review of the Literature on Light Flicker: Ergonomics, Biological Attributes, Potential Health Effects, and Methods in which Some LED Lighting May Introduce Flicker, Tech. report, IEEE Standards P1789, IEEE Press, Piscataway, NJ, 2010. Available online at http://grouper.iee.org/groups/1789

[2] C. Aguerrebere, J. Delon, Y. Gousseau, and P. Musé, Study of the Digital Camera Acquisition Process and Statistical Modeling of the Sensor Raw Data, Tech. report hal-00733538v1, HAL Archives, 2012. Available online at https://hal.archives-ouvertes.fr/file/index/docid/733538/filename/camera_ model.pdf

[3] F.J. Anscombe, The transformation of Poisson binomial and negative-binomial data, Biometrika, 35 (1948), pp. 246-254.

Copyright (C) by SIAM. Unauthorized reproduction of this article is prohibited. 
[4] L. Azzari AND A. FoI, Gaussian-Cauchy mixture modeling for robust signal-dependent noise estimation, in Proceedings of the International Conference on Acoustics, Speech and Signal Processing (ICASSP), Florence, Italy, 2014, pp. 5357-5361.

[5] L. Azzari AND A. FoI, Indirect estimation of signal-dependent noise with nonadaptive heterogeneous samples, IEEE Trans. Image Process., 23 (2014), pp. 3459-3467.

[6] F.R. Boddekke, Quantitative Fluorescence Microscopy, Ph.D. thesis, Technische Universiteit Delft, Delft, The Netherlands, 1999.

[7] J. Boulanger, C. Kervrann, P. Bouthemy, P. Elbau, J.-B. Sibarita, and J. Salamero, Patchbased nonlocal functional for denoising fluorescence microscopy image sequences, IEEE Trans. Med. Imaging, 29 (2010), pp. 442-454.

[8] A. Buades, B. Coll, And J.M. Morel, A review of image denoising algorithms, with a new one, SIAM J. Multiscale Model. Simul., 4 (2005), pp. 490-530.

[9] T. Buades, Y. Lou, J.-M. Morel, And Z. Tang, A note on multi-image denoising, in Proceeding of the International Workshop on Local and Non-Local Approximation in Image Processing, Tuusalu, Finland, 2009, pp. 1-15.

[10] R. Cai, J.F.G. Cobben, J.M.A. Myrzik, J.H. Blom, and W.L. Kling, Flicker responses of different lamp types, IET Generation, Transmission \& Distribution, 3 (2009), pp. 816-824.

[11] M. Colom, A. Buades, And J.-M. Morel, Nonparametric noise estimation method for raw images, J. Opt. Soc. Amer. A, 31 (2014), pp. 863-871.

[12] D.R. Cox, Some statistical methods connected with series of events, J. Roy. Statist. Soc. Ser. B, 17 (1955), pp. 129-164.

[13] D.J. Daley And D. Vere-Jones, An introduction to the theory of point processes, vol. 1, Springer, 2nd ed., 2003.

[14] J. Delon, Midway image equalization, J. Math. Imaging Vis., 21 (2004), pp. 119-134.

[15] J. Delon And A. Desolneux, Stabilization of flicker-like effects in image sequences through local contrast correction, SIAM J. Imaging Sci., 3 (2010), pp. 703-734.

[16] S. Delpretti, F. Luisier, S. Ramani, T. Blu, And M. Unser, Multiframe sure-let denoising of timelapse fluorescence microscopy images, in Proceedings of the IEEE International Symposium on Biomedical Imaging (ISBI), Paris, 2008, pp. 149-152.

[17] B. Ellingwood and A. Tallin, Structural serviceability: Floor vibrations, J. Struct. Engrg., 110 (1984), pp. 401-418.

[18] H. Faraji and W.J. MacLean, CCD noise removal in digital images, IEEE Trans. Image Process., 15 (2006), pp. 2676-2685.

[19] A. FoI, Clipped noisy images: Heteroskedastic modeling and practical denoising, Signal Process., 89 (2009), pp. $2609-2629$.

[20] A. Foi, S. Alenius, V. Katvonik, and K. Egiazarian, Noise measurement for raw-data of digital imaging sensors by automatic segmentation of non-uniform targets, IEEE Sensors J., 7 (2007), pp. $1456-1461$.

[21] A. Foi, M. Trimeche, V. Katkovnik, and K. Egiazarian, Practical Poissonian-Gaussian noise modeling and fitting for single-image raw-data, IEEE Trans. Image Process., 17 (2008), pp. 17371754 .

[22] M. GRÉDiac AND F. SuR, Effect of sensor noise on the resolution and spatial resolution of displacement and strain maps estimated with the grid method, Strain, 50 (2014), pp. 1-27.

[23] G.E. Healey and R. Kondepudy, Radiometric CCD camera calibration and noise estimation, IEEE Trans. Pattern Anal. Mach. Intell., 16 (1994), pp. 267-276.

[24] F. Hild And S. Roux, Comparison of local and global approaches to digital image correlation, Experimental Mech., 52 (2012), pp. 1503-1519.

[25] H.T. НyтTi, Characterization of digital image noise properties based on RAW data, in Proceedings of Image Quality and System Performance III, Proc. SPIE 6059, San Jose, CA, 2006, 6059A.

[26] L. IsSERLIS, On a formula for the product-moment coefficient of any order of a normal frequency distribution in any number of variables, Biometrika, 12 (1918), pp. 134-139.

[27] A. Jezierska, C. Chaux, J.-C. Pesquet, H. Talbot, and G. Engler, An EM approach for timevariant Poisson-Gaussian model parameter estimation, IEEE Trans. Signal Process., 62 (2014), pp. 17-30.

Copyright (C) by SIAM. Unauthorized reproduction of this article is prohibited. 
[28] A. Jezierska, J.-C. Pesquet, H. Talbot, and C. Chaux, Iterative Poisson-Gaussian noise parametric estimation for blind image denoising, in Proceedings of the IEEE International Conference on Image Processing (ICIP), Paris, 2014, pp. 2819-2823.

[29] X. Jin, Z. XU, AND K. HirakaWa, Noise parameter estimation for Poisson corrupted images using variance stabilization transforms, IEEE Trans. Image Process., 23 (2014), pp. 1329-1339.

[30] D.S. KePshire, Sub-surface diffuse fluorescence tomography: System development and feasability studies, Master's thesis, Thayer School of Engineering, Dartmouth College, Hanover, NH, 2006.

[31] M. Lebrun, M. Colom, A. Buades, And J.-M. Morel, Secrets of image denoising cuisine, Acta Numer., 21 (2012), pp. 475-576.

[32] C. Liu, R. Szeliski, S.B. Kang, C.L. Zitnick, and W.T. Freeman, Automatic estimation and removal of noise from a single image, IEEE Trans. Pattern Anal. Mach. Intell., 30 (2008), pp. 299-314.

[33] X. Liu, M. Tanaka, and M. Okutomi, Practical signal-dependent noise parameter estimation from a single noisy image, IEEE Trans. Image Process., 23 (2014), pp. 4361-4371.

[34] Z. Liu, L. Yuan, X. Tang, M. Uyttendaele, and J. Sun, Fast burst images denoising, ACM Trans. Graphics, 33 (2014), pp. 232:1-232:9.

[35] R.W. Lohman, Neon tube characteristics, Elec. Engrg., 52 (1933), pp. 304-307.

[36] M. Makitalo And A. Foi, Optimal inversion of the generalized Anscombe transformation for PoissonGaussian noise, IEEE Trans. Image Process., 22 (2013), pp. 91-103.

[37] M. Makitalo AND A. FoI, Noise parameter mismatch in variance stabilization, with an application to Poisson-Gaussian noise estimation, IEEE Trans. Image Process., 23 (2014), pp. 5348-5359.

[38] P. Milanfar, A tour of modern image filtering: New insights and methods, both practical and theoretical, IEEE Signal Process. Mag., 30 (2013), pp. 106-128.

[39] J. Molimard and L. Navarro, Uncertainty on fringe projection technique: A Monte-Carlo-based approach, Optics Lasers Engrg., 51 (2013), pp. 840-847.

[40] F. Murthagh, J.L. Starck, And A. Bijaoui, Image restoration with noise suppression using a multiresolution support, Astronom. Astrophys., 112 (1995), pp. 179-189.

[41] G.W. Oehlert, A note on the Delta method, Amer. Statist., 46 (1992), pp. 27-29.

[42] S. PYATYKH AND J. HeSSER, Image sensor noise parameter estimation by variance stabilization and normality assessment, IEEE Trans. Image Process., 23 (2014), pp. 3990-3998.

[43] S. Ramani, C. Vonesch, And M. Unser, Deconvolution of $3 d$ fluorescence micrographs with automatic risk minimization, in Proceedings of the IEEE International Symposium on Biomedical Imaging (ISBI), Paris, 2008, pp. 732-735.

[44] P. Roudot, C. Kervrann, J. Boulanger, and F. Waharte, Noise modeling for intensified camera in fluorescence imaging: Application to image denoising, in Proceedings of the IEEE International Symposium on Biomedical Imaging (ISBI), San Francisco, 2013, pp. 600-603.

[45] P. Roudot, C. Kervrann, F. Waharte, and J. Boulanger, Lifetime map reconstruction in frequencydomain fluorescence lifetime imaging microscopy, in Proceedings of the International Conference on Image Processing (ICIP), Orlando, 2012, pp. 2537-2540.

[46] Sensicam QE-1288 Data, Tech. report, PCO Imaging, Kelheim, Germany, 2005. Available online at http://www.pco.de/fileadmin/user_upload/db/products/datasheet/sensicam_qe_lgdlm_1288sheet_ 0510.pdf

[47] Standard 1288, Standard for Characterization of Image Sensors and Cameras, Release 3.0, Tech. report, European Machine Vision Association (EMVA), Barcelona, 2010. Available online at http://www. emva.org/cms/upload/Standards/Stadard_1288/EMVA1288-3.0.pdf

[48] S. Sun, M. Grédiac, E. Toussaint, J.-D. Mathias, and N. Mati-Baouche, Applying a full-field measurement technique to characterize the mechanical response of a sunflower-based biocomposite, Experimental Mech., to appear. Published online Feburary 20, 2015; doi: 10.1007/s11340-015-9988-1.

[49] F. Sur AND M. GrÉdiac, Sensor noise measurement in the presence of a flickering illumination, in Proceedings of the IEEE International Conference on Image Processing (ICIP), Paris, 2014, pp. 1763-1767.

[50] F. Sur And M. GréDiac, Sensor noise modeling by stacking pseudo-periodic grid images affected by vibrations, IEEE Signal Process. Lett., 21 (2014), pp. 432-436.

[51] F. SuR AND M. GRÉDIAC, Towards deconvolution to enhance the grid method for in-plane strain measurement, Inverse Probl. Imaging, 8 (2014), pp. 259-291.

Copyright (C) by SIAM. Unauthorized reproduction of this article is prohibited. 
[52] F. Sur AND M. Grédiac, Measuring the Noise of Imaging Sensors in the Presence of Vibrations and Illumination Flickering: Modeling, Algorithm, and Experiments, Research Report 8672, INRIA, Paris, 2015.

[53] F. SuR AND M. GRÉDIAC, On noise reduction in strain maps obtained with the grid method by averaging images affected by vibrations, Optics Lasers Engrg., 66 (2015), pp. 210-222.

[54] T.H. Thai, R. Cogranne, and F. Retraint, Camera model identification based on the heteroscedastic noise model, IEEE Trans. Image Process., 23 (2014), pp. 250-263.

[55] M.L. Uss, B. Vozel, V.V. Lukin, And K. CheHdi, Image informative maps for component-wise estimating parameters of signal-dependent noise, J. Electron. Imaging, 22 (2013), 013019.

[56] S.B. VARdeman And C.-S. LEE, Likelihood-based statistical estimation from quantized data, IEEE Trans. Instrumentation and Measurement, 54 (2005), pp. 409-414.

[57] B. WAEgLI, Investigations into the noise characteristics of digitized aerial images, Int. Arch. for Photogr. and Remote Sensing, 32 (1998), pp. 341-348.

Copyright $($ b by SIAM. Unauthorized reproduction of this article is prohibited. 